\title{
Rapid and specific detection of Salmonella infections using chemically modified nucleic acid probes
}

Isabel Machado, Victoria Garrido, Luiza Hernandez, J uliana Botero, Nora Bastida, Beatriz San-Roman, Maria-J esus Grillo and Frank Hernandez

The self-archived postprint version of this journal article is available at Linköping University Institutional Repository (DiVA):

http:/ / urn.kb.se/ resolve?urn=urn:nbn:se:liu:diva-154523

N.B.: When citing this work, cite the original publication.

Machado, I., Garrido, V., Hernandez, L., Botero, J ., Bastida, N., San-Roman, B., Grillo, M., Hernandez, F., (2019), Rapid and specific detection of Salmonella infections using chemically modified nucleic acid probes, Analytica Chimica Acta, 1054, 157-166. https:// doi.org/ 10.1016/j.aca.2018.12.027

Original publication available at:

https:// doi.org/ 10.1016/j.aca.2018.12.027

Copyright: Elsevier (12 months)

http:// www.elsevier.com/

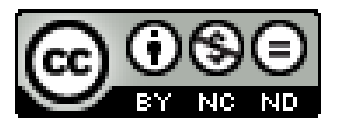




\section{Rapid and specific detection of Salmonella infections using chemically modified nucleic acid probes}

Isabel Machado $\# 1$, Victoria Garrido $\# 2$, Luiza I. Hernandez ${ }^{1,3}$, Juliana Botero ${ }^{1}$, Nora Bastida ${ }^{1}$, Beatriz SanRoman², María-Jesús Grilló ${ }^{2}$ and Frank J. Hernandez ${ }^{\star 4,5}$

${ }^{1}$ SOMAprobes, Science and Technology Park of Gipuzkoa, Donostia-San Sebastian, 20009, Spain.

${ }^{2}$ Animal Health Research Group, Institute of Agrobiotechnology, CSIC-UPNa-Gobierno de Navarra, Mutilva, 31192, Spain.

${ }^{3}$ Department of Clinical and Experimental Medicine, Linköping University,Linköping, 58185, Sweden

${ }^{4}$ Department of Physics, Chemistry and Biology, Linköping University, Linköping, 58185, Sweden

${ }^{5}$ Wallenberg Centre for Molecular Medicine (WCMM), Linköping University, Linköping, Sweden

\# Co-first authors

*To whom correspondence should be addressed. frank.hernandez@liu.se

\section{ABSTRACT}

Salmonella is a leading source of bacterial foodborne illness in humans, causing gastroenteritis outbreaks with bacteraemia occurrences that can lead to clinical complications and death. Eggs, poultry and pig products are considered as the main carriers of the pathogenic Salmonella for humans. To prevent this relevant zoonosis, key changes in food safety regulations were undertaken to improve controls in the food production chain. Despite these measures, large outbreaks of salmonellosis were reported worldwide in the last decade. Thus, new strategies for Salmonella detection are a priority for both, food safety and public health authorities. Such detection systems should provide significant reduction in diagnostic time (hours) compared to the currently available methods (days). Herein, we report on the discovery and characterization of nucleic acid probes for the sensitive and specific detection of live Salmonella within less than 8 hours of incubation. We are the first to postulate the nuclease activity derived from Salmonella as biomarker of infection and its utility to develop innovative detection strategies. Our results have shown the screening and identification of two oligonucleotide sequences (substrates) as the most promising probes for detecting Salmonella - Sal-3 and Sal-5. The detection limits for both probes were determined with the reference Salmonella Typhimurium (STM 1) and Salmonella Enteritidis (SE 1) cultures. Sal-3 has reported LOD values around $10^{5} \mathrm{CFU} \mathrm{mL}^{-1}$ for STM 1 and $10^{4} \mathrm{CFU} \mathrm{mL} \mathrm{m}^{-1}$ for SE 1 , while Sal-5 proves to be a slightly better probe, with LODs of $10^{4}$ CFU $\mathrm{mL}^{-1}$ for STM 1 and $10^{4} \mathrm{CFU} \mathrm{mL} \mathrm{m}^{-1}$ for SE 1 . Both selected probes have shown the capability to recognize 49 out of 51 different Salmonella serotypes tested in vitro and the most frequent serotypes in porcine mesenteric lymph nodes as a standard sample used in fattening-pig salmonellosis baseline studies. Notably, our results showed 100\% correlation between nuclease detection and the PCR-InvA or ISO-6579 standard method, underlining the great potential of this innovative nucleic acids technology to be implemented as a rapid method for food safety testing. 


\section{KEYWORDS}

Salmonella, nucleic acid probes, detection system, nucleases.

\section{INTRODUCTION}

Salmonella is an important leading cause of human bacterial foodborne illness from animal origin. Poultry and pig products are considered as the main carriers of the pathogenic Salmonella, but the pathogen can be found in most foods such as meats, eggs, milk, cheese, vegetables and ready-to-eat products, including nuts and seeds $[1,2]$. While these animals often present asymptomatic infections, salmonellosis in humans produces gastroenteritis and high risk of systemic infections, which are life threatening [3-5]. In Europe, more than 100,000 cases of human salmonellosis are occurring every year, according to the European Food Safety Authority (EFSA) reports [6]. In the USA, this value increases to 1.2 millions of infections, causing around 19,000 hospitalizations and 450 deaths per year, according to the Food and Drug Administration (FDA) reports [7, 8]. Thus, reduction of salmonellosis is a stated aim worldwide.

The detection of Salmonella is generally performed by microbiological culture techniques [9-12] that are confirmatory but they suffer from low sensitivity, expensive, labour intensive, require trained personnel and are time consuming, taking from days to weeks to confirm the presence or absence of Salmonella. Alternative methods based on PCR and immunoassays $[13,14]$ have been developed, however these methods could detect DNA from dead bacteria (e.g. after pasteurization) given false-positive results and/or require an earlier culture phase that delay up to several days confirmatory results within the sensitivity criteria stipulated in the regulations (). These times are clearly incompatible with the rapid speed at which food moves through the production and distribution chain "from farm to fork", and at which human salmonellosis foodborne outbreaks usually appear. Accordingly, reliable alternative methods are still a clear priority for the food industry and at the clinical level. The speed of the diagnostic method is important not only for preventing clinical complications, but also for identifying the origin of the outbreak and further efficient removing the contaminated products from the market.

Herein, we report for the first time the development of a detection system based on chemically modified nucleic acids for the specific targeting of Salmonella using its global nuclease activity as biomarker.

Figure 1 describes the detection strategy that was implemented in this study. Importantly, we used chemically modified oligonucleotides flanked at the 5'-end with a fluorophore (FAM) and at the 3'-end with a quencher (TQ2). In this strategy, the reporter molecule (FAM) is quenched by the close proximity with the quencher, and only upon degradation of the oligonucleotide probe by a specific nuclease, the FAM and quencher moieties diffuse away, increasing the initial distance. This allows the FAM moiety to recover its functionality as reporter molecule, and the nuclease activity event can be recorded by fluorescence measurements. Previously, it was reported the utility of a single nuclease activity (Micrococcal Nuclease) for detecting Staphylococcus aureus bacterial infections in animal models, demonstrating the potential of this technology for targeting bacteria where a given nuclease works as biomarker [15]. In the present study, we sought to detect the global nuclease activity (blueprint) derived 
from Salmonella in a specific and sensitive manner using nucleic acid oligonucleotides as substrates. This methodology allows the identification of unknown nucleases as biomarkers for detection purposes. A graphical representation of the screening process is shown in Figure S1. First, we have screened the nuclease activity profile in bacterial cultures of Salmonella enterica subsp. enterica serotypes Typhimurium and Enteritidis (S. Typhimurium and S. Enteritidis, ahead) using nucleic acid libraries containing several sequences and chemical modifications. Next, based on the preliminary data obtained from this screening, we have designed and tested additional sequences. As result, we have identified two optimal oligonucleotide probes with high specificity and sensitivity for Salmonella that were validated in a blind study using porcine lymph nodes tissue. Importantly, these probes were able to identify with $100 \%$ accuracy the presence of Salmonella in these tissues, when compared with the standard microbiological methods and PCR. In this work, we have demonstrated the applicability of chemically modified nucleic acid probes for detecting Salmonella in a relevant environment sample, underlining the great potential of this technology to be implemented as an alternative method for food safety testing.

\section{MATERIAL AND METHODS}

\subsection{Materials}

\subsubsection{Solutions and culture media}

Two types of Phosphate Buffered Saline (PBS, $\mathrm{pH}$ 7.4) with $\mathrm{MgCl}_{2}$ and $\mathrm{CaCl}_{2}(\mathrm{PBS}+/+$ ) and without this supplement (PBS -/-) were purchased from Gibco (Life Technologies, Spain). Ethylene-diaminetetra-acetic acid (EDTA, CAS number: 60-00-4, pH 8.0), Tris-EDTA, 1x Solution (TE, CAS number: 38641-82-6, pH 8.0) and Nitro-triacetic acid (NTA, CAS number: 139-13-9) were purchased from ThermoFisher Scientific (Madrid, Spain). Ethylene-glycol-bis(2-aminoethylether)-N (EGTA, CAS number: 67-42-5), Magnesium chloride hexahydrate (CAS number: 7791-18-6), Calcium chloride (CAS number: 1043-52-4), Zinc chloride (CAS number: 7646-85-7), Manganese (II) chloride (CAS number: 7773-01-5) and Copper (II) acetate (CAS number: 142-71-2) were purchased from Sigma-Aldrich (Madrid, Spain).

Buffered Peptone Water (BPW), Luria-Bertani Agar (LA), Tryptone Soya Agar (TSA), Luria-Bertani (LB), and Tryptic Soy Broth (TSB) culture media were purchased in Laboratorios Conda SL (Spain). Bacterial strains were stored frozen at $-20^{\circ} \mathrm{C}$ in $10 \%$ skimmed milk (Laboratorios Conda, Spain) supplemented with $1 \%$ lactose (Laboratorios Conda, Spain) until their use.

Agarose gel, TAE buffer and Midori Green DNA staining were also purchased in Laboratorios Conda (Spain).

\subsubsection{Bacterial strains}

A total of 93 bacterial strains (Table S1) were selected from the bacterial culture collection of the Institute of Agrobiotechnology (IdAB, Navarra, Spain), including 11 S. Typhimurium (STM1 - STM11), 11 S. Enteritidis (SE1 - SE11), 51 different Salmonella spp. serotypes (S1 - S51) and 20 non-Salmonella 
strains (NS1 - NS20) belonging to 12 different genera. All these strains were previously isolated from animal or food samples and characterized in the IdAB, and confirmed by sero- and geno- typing in the National Reference Centre for Animal Salmonellosis (Algete, Madrid, Spain) or in the Health Institute ISC Carlos III (Madrid, Spain) [16, 17]. All the strains were stored at $-20^{\circ} \mathrm{C}$ in $10 \%$ skimmed milk.

\subsubsection{BPW-MLN samples and PCR-InvA reactions}

Fattening-pig mesenteric lymph nodes (BPW-MLN) samples were previously cultured in BPW $\left(37^{\circ} \mathrm{C}\right.$, 24h) as recommended by the ISO 6579 standard method [18] and 1-mL aliquots (BPW-MLN) were kept at $-20^{\circ} \mathrm{C}$ in the IdAB collection. Thirty of these BPW-MLN samples previously characterized by ISO 6579 and PCR-InvA $[14,19]$ methods (Table S3 and Figure S3, respectively) were evaluated by nuclease activity assay in a blind experiment without knowing the status of Salmonella infection.

\subsubsection{Oligonucleotide probes synthesis and purification}

The oligonucleotide sequences and names of the probes used in this study are described in Table S2. All probes were synthesized and purified at Biomers.net (Germany) with fluorescein amidite (FAM) fluorophore at the 5'-end and the tide quencher 2 acid (TQ2) at the 3'- end. For this, a standard method of solid-phase phosphor-amidite chemistry was used, followed by high-performance liquid chromatography (HPLC) purification. The probe identities were confirmed with matrix-assisted laser desorption ionization mass spectrometry (MALDI-MS). The purity of the probes, as assessed with HPLC analysis, is typically greater than $95 \%$.

\subsection{Methods}

\subsubsection{Supernatants preparation for nuclease assay}

To prepare pure culture supernatants, frozen bacteria were pre-cultured in LA or TSA plates (24 h, $37^{\circ} \mathrm{C}$ ) and then one individual colony was transferred to LB or TSB and incubated overnight, at $37^{\circ} \mathrm{C}$. The cultures obtained were diluted 1:500 in fresh LB or TSB and cultured $\left(24 \mathrm{~h}, 37^{\circ} \mathrm{C}\right)$ under shaking at $200 \mathrm{rpm}$. Each culture was then centrifuged $(6,000 \mathrm{~g}, 20 \mathrm{~min})$ and the supernatant was removed and kept at $4^{\circ} \mathrm{C}$ until its use.

On the other hand, BPW-MLN aliquots were thawed and centrifuged $(6,000 \mathrm{~g}, 20 \mathrm{~min})$ for supernatant collection.

For biosecurity purposes, each supernatant was filtered in a $0.2 \mu \mathrm{m}$ Millipore ${ }^{\circledR}$ (Sarsted). To confirm the absence of bacteria, $100 \mu \mathrm{L}$ of each filtrate was plated on TSA and incubated at $37^{\circ} \mathrm{C}$. After $48 \mathrm{~h}$, the bacterial growth was evaluated. The supernatants with negative growth in plates (no bacteria) were further stored at $4^{\circ} \mathrm{C}$ until use.

\subsubsection{Nuclease activity assays}


The nuclease activity assays were performed using the standard conditions previously reported [15]. Specifically, for each reaction, $9 \mu \mathrm{L}$ of sample (i.e. culture supernatants of Salmonella, other bacteria or mesenteric lymph nodes, control medium or buffer) were mixed with $1 \mu \mathrm{L}$ (50 pmol, nuclease substrate) of the oligonucleotide probe to be tested and incubated at $37^{\circ} \mathrm{C}$, for $1 \mathrm{~h}$. Thereafter, the reaction was stopped by adding $295 \mu \mathrm{L}$ of PBS -/- supplemented with $10 \mathrm{mM}$ EDTA. Next, $95 \mu \mathrm{L}$ of each sample was loaded in triplicates into 96-well black plates (96F non-treated black microwell plate, Thermo Scientific). Fluorescence intensity was measured with a fluorescence microplate reader (Synergy HT, BioTek) using the filter settings for FAM (excitation/emission 494/521 nm). Three independent experiments were performed with each sample. The results were expressed as the mean $\pm \mathrm{SD}(\mathrm{n}=3)$ of fluorescence intensity in arbitrary units (a.u.).

\subsubsection{Screening of oligonucleotide probes in Salmonella and other bacterial cultures}

A first screening was performed by using 12 oligonucleotide probes (named P1 to P12, Table S2), classified in three categories: i) DNA and RNA natural probes; ii) 2'-fluoro probes [named as 2'-F] and its Pyrimidine (Pyr) and Purine (Pur) derivatives; and iii) 2'-O-methyl probes [named as 2'-OMe] and its Pyr and Pur derivatives (Table S2). The ability of these 12 probes to detect nuclease activity of Salmonella spp. were assessed in culture supernatants of STM 1, SE 1 and three other common ubiquitous bacteria (i.e. Escherichia coli NS 1, Staphylococcus aureus NS 16 and Streptococcus pyogenes NS 19 - Table S1), under standard conditions. LB supernatant was used as control.

Second, the probe that better detected nucleases of Salmonella in contrast to the non-Salmonella strains was further used as "parental-probe" to design other 12 "derivative-probes", named as Sal-1 to Sal-12 (Table S2). To assess the ability of the "derivative-probes" to detect the nucleases produced by S. Typhimurium and S. Enteritidis, these 12 probes were tested with supernatants of STM 1, SE 1 and other 20 strains of these two serotypes (named STM 2 to STM 11 and SE 2 to SE 11, Table S1). The parental-probe was used as control.

Third, two "candidate-probes" were selected according to their capability to detect higher nuclease activity in all the STM and SE strains tested. The specificity and sensitivity of these candidate-probes to detect Salmonella spp. were further determined.

\subsubsection{Specificity of the candidate-probes under different conditions}

The specificity of the candidate-probes to detect Salmonella spp. nuclease activity was assessed in an initial experiment with the 20 NS strains supernatants (Table S1), under conventional conditions (i.e. addition of PBS $+/+$ and incubation $37^{\circ} \mathrm{C}, 1 \mathrm{~h}$ ). STM 1 and SE 1 supernatants and the Pyr 2'-OMe RNA parental-probe were used as controls.

The lack of specificity observed was circumvented by the optimization of the nuclease activity conditions, considering, first, the optimal time of incubation and, second, the use of different chemical conditions that could allow the best discrimination between Salmonella and non-Salmonella strains. The optimal time of incubation was determined by mixing Sal-3 or Sal- 5 candidate-probes with STM 1 or SE 1 supernatants as detailed for standard conditions (see above) and further incubation at $37^{\circ} \mathrm{C}$ for $7.5 \mathrm{~min}$, 
$15 \mathrm{~min}, 30 \mathrm{~min}, 60 \mathrm{~min}$ and $120 \mathrm{~min}$. At each selected point-time, the reaction was stopped and processed as stated above. Thereafter, different chemical conditions with chelators and divalent cations were analysed by mixing $1 \mu \mathrm{L}$ ( $50 \mathrm{pmol}$ ) of the correspondent Sal-3 and Sal- 5 probes with a suspension of $8 \mu \mathrm{L}$ of STM 1 or SE 1 supernatants plus $1 \mu \mathrm{L}$ of $50 \mathrm{mM}$ of EDTA, EGTA or NTA chelators or $1 \mu \mathrm{L}$ of $\mathrm{Mg}^{2+}, \mathrm{Ca}^{2+}, \mathrm{Zn}^{2+}, \mathrm{Mn}^{2+}$ and $\mathrm{Cu}^{2+}$ divalent cations. After incubation $\left(37^{\circ} \mathrm{C}, 1 \mathrm{~h}\right)$, the reaction was stopped and processed as before (see above).

The final assessment of specificity was performed using the optimized conditions (i.e. addition of EGTA chelating agent and incubation $37^{\circ} \mathrm{C}, 15 \mathrm{~min}$ ) for detecting nucleases in the same $20 \mathrm{NS}$ supernatants used in the initial experiment.

\subsubsection{Sensitivity of the candidate-probes to detect different Salmonella spp.}

To determine the sensitivity of Sal-3 and Sal-5 to detect not only STM and SE but also other Salmonella spp, a wide panel $(n=51)$ of different Salmonella serotypes was selected from the cultures collection of IdAB (Table S1) and tested under the optimized conditions (see above).

\subsubsection{Limit of detection of nuclease activity in pure bacterial cultures}

The limit of detection (LOD) of the technique based on the nuclease activity was determined using the in vitro growth curves STM 1 and SE 1 pure culture supernatants, with Parental, Sal-3 and Sal-5 candidate-probes. For this, the Salmonella stock was cultured (at $37^{\circ} \mathrm{C}, 24 \mathrm{~h}$ ) in LA plates and, then, harvested and adjusted by spectrophotometry $\left(\mathrm{OD}_{600 \mathrm{~nm}}=0.160\right)$ to $\approx 2 \times 10^{8} \mathrm{CFU} \mathrm{mL}^{-1}$ in BPW. Serially ten-fold dilutions from this suspension were prepared down to $\approx 1 \times 10^{2} \mathrm{CFU} \mathrm{mL}^{-1}$ in a final volume of 60 $\mathrm{mL}$ (prepared by mixing $6 \mathrm{~mL}$ of $\mathrm{a} \approx 10^{3} \mathrm{CFU} \mathrm{mL} \mathrm{L}^{-1}$ and $54 \mathrm{~mL}$ of BPW) and the bacterial growth curves were determined at $0,2,4,6,8,10$ and $24 \mathrm{~h}$ after incubation at $37^{\circ} \mathrm{C}$, by triplicate viable bacterial counting (number of CFU mL $\mathrm{mL}^{-1}$ ) at each selected time point. Each sample was analysed by triplicate. The individual number of CFU mL-1 was logarithmically transformed and the results were expressed as the mean $\pm S D(n=3) \log _{10} C F U \mathrm{~mL}^{-1}$.

Moreover, supernatants of each point-time sampling were collected by centrifugation (6,000 $\mathrm{g}, 20 \mathrm{~min})$ and used in the nuclease assay with Sal- 3 and Sal- 5 candidate-probes under standard conditions $\left(37^{\circ} \mathrm{C}\right.$, $1 \mathrm{~h})$. The Pyr 2'-OMe RNA parental-probe was used as control. The nuclease activity was measured by fluorescence intensity and the results were normalized for the maximum value obtained at $24 \mathrm{~h}$ of bacterial growth. The LOD for the 3 probes (Parental, Sal-3 and Sal-5) was calculated according to the formula: LOD $=3^{\star} S y^{*} S / o p e^{-1}$ as was previously reported [20], where Sy is the standard deviation of the response curve and slope represents the slope of the linear fit (Figures $\mathbf{4 a}$ and $\mathbf{4 b}$ ). This analysis was carried out using GraphPad Prism 5.

2.2.7. Efficacy of the candidate-probes for detecting Salmonella spp. nucleases in BPW-MLN field samples 
The sensitivity of Sal-3 and Sal-5 candidate-probes were determined in a blind nuclease activity experiment with the 30 BPW-MLN supernatants selected (see above), by using the optimal conditions previously defined (i.e. addition of EGTA chelator and incubation $15 \mathrm{~min}$ ). Besides the ISO 6579 previous classification [17], the presence of Salmonella in BPW-MLN samples was checked by a PCRInvA previously described [14]. Briefly, the PCR-InvA were performed with the DNA extracted from 0.5 $\mathrm{mL}$ of a thawed BPW-MLN aliquot and the amplification of the expected 229-bp DNA fragments were revealed by conventional electrophoresis in a $1 \%(\mathrm{w} / \mathrm{v})$ agarose gel with TAE buffer (Laboratorios Conda, Spain), Midori Green staining and visualized in a UV transilluminator. STM1 (positive control) and $E$. coli and sterile ultra-pure water (MilliQ) (negative controls) were used as controls.

\section{RESULTS}

\subsection{Screening of nuclease activity in Salmonella cultures}

We sought to identify specific nuclease activity derived from Salmonella by designing oligonucleotide substrates containing different sequences and chemical modifications. We began our screening with 12 different libraries of DNA and RNA, 2'-fluoro and 2'-O-methyl probes and its derivatives that were incubated with supernatants of the target bacteria (Salmonella STM1 and SE 1) or three non-target $E$. coli, S. aureus and S. pyogenes, as controls. Figure 2 shows the preference of the nuclease activity found in Salmonella and control cultures for each tested probe. The DNA and RNA natural probes have reported nuclease activity for both Salmonella and control bacteria, suggesting the presence of multiple nucleases in each of the bacterial cultures. The DNA probes were easily degraded by the $S$. aureus cultures, which are consistent with previous report on the activity of Micrococcal Nuclease secreted by this bacterium [15]. On the other hand, the RNA probe was slightly better degraded by Salmonella cultures compared to the control bacteria, at the level or higher than S. aureus (Figure 2). These results suggest that Salmonella cultures have a preference for the RNA over the DNA oligonucleotide probes tested in this study, and this could indicate the presence of active RNases in the cultures.

Subsequently, both RNA and DNA probe sequences were fully or partially modified by incorporating the two well established 2'-fluoro and 2'-O-methyl chemical modifications of the nucleosides during the oligonucleotide synthesis (Table S2). Thus, it is expected that the resulting chimeras confer additional features of resistance and/or specificity for a target nuclease activity associated to Salmonella cultures. Indeed, we observed that the five 2'-fluoro modified probes were better digested by the control nonspecific bacteria than Salmonella cultures, suggesting that 2'-fluoro chemistry is a less suitable modification for the identification of Salmonella nucleases (Figure 2). More specifically, Salmonella cultures have reported a lower signal for the fully 2'-fluoro modified probe, as well as for the Pyr-2'-FDNA and Pur-2'-F-DNA chimeras. Interestingly, the Pyr-2'-F-RNA and Pur-2'-F-RNA chimeras have shown only slightly lower fluorescent values compared to the controls, indicating that RNA containing probes are more susceptible to specific degradation by Salmonella nucleases.

Next, we tested the 2'-O-methyl modified probes. We detected that the fully 2'-O-methyl (All 2'-OMe) sequence is the most resistant probe for all bacterial cultures tested, including Salmonella cultures, this finding being in good agreement with the previous report [15]. On the other hand, the 2'-O-methyl DNA 
chimeras (Pyr-2'-OMe-DNA and Pur-2'-OMe-DNA) generated the highest fluorescence intensity values, thus highest nuclease activity for the $S$. aureus cultures, and limited nuclease activity for Salmonella cultures. In contrast, very promising results were observed for the 2'-O-methyl RNA chimeras, where Pyr-2'-OMe-RNA probe has shown the best ability for discriminating between Salmonella and the other bacterial cultures (Figure $\mathbf{2}$ - dashed line rectangle). These results are in agreement with the previous findings, regarding the susceptibility of RNA containing probes to degradation by Salmonella nucleases. We also observed a remarkable difference between the Pyr-2'-OMe-DNA and Pyr-2'-OMe-RNA probes, most probably attributed to the presence of the DNA and RNA purines, respectively. This observation suggests that the three RNA purines in the Pyr-2'-OMe-RNA sequence (see Table S2) are essential for targeting Salmonella nucleases. Thus, we sought to further optimize the probe by modifying the number and sequence of these three RNA purines, so to obtain a specific probe for Salmonella.

\subsection{Design and characterization of an optimal probe for Salmonella}

Based on the successful identification of the parental-probe (Pyr-2'-OMe-RNA) as candidate probe to identify Salmonella, we were motivated to design additional oligonucleotide "derivative-probes" by changing the three purines RNA nucleotides present in the parental-probe. To do so, the 12-mer sequence of the parental-probe was modified to evaluate the effect wherein the incorporation of a single adenosine or guanosine or several purines could improve the probe sensitivity when compared to the parental-probe. With this in mind, 12 new "derivative-probes" (Sal-1 to Sal-12 - Table S2) were designed to be tested under the standard conditions of the initial screening. Figure 3 shows the degradation efficiency of all probes using 11 cultures of Salmonella Typhimurium (Figure 3a) and 11 cultures of Salmonella Enteritidis (Figure $\mathbf{3 b}$ ). The degradation efficiency of the parental-probe with each supernatant was used as base line for evaluating the activity of the new derivative-probes. Interestingly, a variety of probe degradation profiles were observed.

Sal-1 and Sal-2 were designed by reducing the number of the RNA purines in the parental-probe sequence, from three purines to a single adenosine in Sal-1 and a single guanosine in Sal-2. For both derivative sequences, a clear reduction in probe degradation was observed (Figure 3). Moreover, the fluorescence signal was most drastically reduced in Sal-2, indicating that the presence of guanosine increases probe resistance towards Salmonella nucleases.

Next, we tested the derivative probes Sal-3 and Sal-4, containing two adenosines and two guanosines, respectively. Sal-4 showed a slight improvement compared to Sal-2, but provided less efficient degradation than the parental-probe (Figure 3). In contrast, Sal-3 proved to be the best probe, indicating that adenosine nucleotides are a better substrate for Salmonella nucleases.

Thereafter, we tested the effect of three adenosines incorporation for Sal-5 and three guanosines for Sal-6. As result, the preference of both $S$. Typhimurium and S. Enteritidis cultures for adenosine as substrate was confirmed once more by a more consistent signal detection (Figure 3).

Finally, we tested the combination of three purines by placing adenosines and guanosines at different positions (Sal-7 to Sal-12), however with no further improvement. These results suggest that Salmonella 
nucleases have a remarkable capability for degrading adenosines, and this ability is reduced in the presence of guanosines.

Overall, these data show that probes Sal-3 and Sal-5 (Figure 3 asterisks), which were modified with two and three adenosines, respectively, are the most promising candidates for targeting Salmonella. Accordingly, both candidate-probes were used in the subsequent sensitivity and specificity studies.

\subsection{Specificity of the Salmonella candidate-probes}

To study the specificity of the Sal-3 and Sal-5 candidate-probes to detect exclusively Salmonella, supernatants from 20 different Non-Salmonella (NS) bacteria were analysed, under conventional conditions (i.e. addition of PBS $+/+$ and incubation $37^{\circ} \mathrm{C}, 1 \mathrm{~h}$ ). The parental-probe and STM 1 and SE 1 supernatants were used as controls. As shown in Figure 4a, supernatants of E. coli, K. pneumoniae, and $S$. aureus reported a significant background signal that could limit the applicability of our technology in real samples.

To overcome the cross-reactivity issues, we attempted the optimization of Salmonella probes by modifying different experimental conditions that can modulate the nuclease activity, such as the time of incubation or the use of chelating agents or divalent cations as detailed above. As shown in Figure S2a, no significant differences in fluorescence signal with Sal-3 and Sal-5 were seen between 15 and 120 min. However, the use of EDTA but not other chelators clearly affected the activity of Salmonella nucleases, suggesting the utility of EGTA and NTA to block the nuclease activity of non-specific bacteria (Figure S2b). Regarding the effect of a variety of $\mathrm{Mg}^{2+}, \mathrm{Ca}^{2+}, \mathrm{Zn}^{2+}, \mathrm{Mn}^{2+}, \mathrm{Cu}^{2+}$ divalent cofactors (Figure S2c), we observed that $\mathrm{Mg}^{2+}, \mathrm{Ca}^{2+}$ and $\mathrm{Mn}^{2+}$ were essential cofactors for the nuclease activity. However, this information was not used, since all probes were reconstituted in PBS +/+, i.e. PBS containing $\mathrm{Mg}^{2+}$, $\mathrm{Ca}^{2+}$. Overall, we established that 15-min incubation and the addition of EGTA are optimal conditions to favour the activity of nucleases associated to Salmonella but not those associated to other bacteria.

Next, we repeated the specificity studies using the optimized conditions for Salmonella with the supernatants of the 20 NS bacteria (Figure 4b). Notably, a significant reduction in nuclease activity for all the cross-reactive bacteria was achieved. In conclusion, we have identified a set of optimized conditions that avoid or minimize unwanted nuclease activity, and enhance or maintain the activity for Salmonella target nucleases. These results suggest an earlier and more specific targeting of Salmonella.

\subsubsection{Sensitivity of the candidate probes to detect different Salmonella serotypes}

On the other hand, we have also evaluated 51 different serotype Salmonella strains under the optimized conditions with Sal-3 and Sal-5 probes. Both probes have shown the capability to recognize 49 out of 51 Salmonella serotypes tested (Figure S4a and Figure S4b, respectively). These results confirm that the tested nucleic acid probes were able to identify a significant number of Salmonella serotypes and this is a remarkable feature for any detection system for targeting Salmonella. 


\subsection{Limit of detection for Salmonella candidate probes in pure cultures}

The LOD of nuclease activity by the two candidate-probes and the parental probe was assessed in Salmonella STM1 and SE1 pure culture supernatants obtained at different time points (0 to $24 \mathrm{~h}$ ). For each time point collected, we have first determined the number of bacteria CFU $\mathrm{mL}^{-1}$ and then we have also performed the nuclease activity assay (Figure S5). These panels in Figure S5 show bacterial growth curves in the exponential phase from 2 to $8 \mathrm{~h}$ post-incubation in both STM1 and SE1 strains. Notably, nuclease activity was detected in the supernatants of all the cultures after 6 hours of incubation. The nucleases derived from Salmonella cultures were able to degrade both Sal-3 and Sal- 5 candidateprobes more efficiently when compared to the parental probe. Moreover, the correlation between bacteria counts and nuclease activity has shown to be more linear for Sal-3 and Sal-5 probes, suggesting that these probes could be better substrates for Salmonella cultures. This correlation allowed us to estimate the number of bacteria and their minimum incubation time that is needed to degrade the nucleic acid probes, by correlating with fluorescence intensity [21-23].

Next, we attempted to calculate the detection limit for each probe by plotting the fluorescence signals ( $Y$ axis) versus the bacteria concentration in CFU $\mathrm{mL}^{-1}$ ( $\mathrm{X}$ axis), using the same samples for both measurements and keeping the time constant for each acquisition point (Figure 5a and Figure 5b) [24, 25]. Based on our screening method described herein (Figure S1), we are selecting our substrates by reducing from millions of possible substrates (12mer length, using 16 different nucleotides $=16^{12}$ possibilities) to a few candidates, but expecting to reach high specificity and sensitivity for developing rapid system for Salmonella detection. With this in mind, the "perfect" nuclease substrate will provide an excellent correlation between bacterial counts and nuclease activity. Our aim in this study is the identification of optimal substrates that provide specificity and a good correlation for bacterial counts and nuclease activity. Figure 5 shows the fitting curves for Salmonella Typhimurium (STM 1) cultures (Figure 5a) and Salmonella Enteritidis (SE 1) cultures (Figure 5b). Then, we have calculated the LOD values for the parental, Sal-3 and Sal-5 probes, as described in the methods section and are depicted in Table S4. The probes Sal-3 and Sal-5 have reported the best LOD values when compared with the parental probe and with a good linearity. Sal-3 has reported LOD values around $10^{5} \mathrm{CFU} \mathrm{mL}^{-1}\left(\mathrm{r}^{2}=\right.$ $0.7771)$ for STM 1 and $10^{4} \mathrm{CFU} \mathrm{mL} \mathrm{m}^{-1}\left(r^{2}=0.8576\right)$ for SE 1 , while Sal-5 proves to be a slightly better probe, with LODs of $10^{4} \mathrm{CFU} \mathrm{mL} \mathrm{mL}^{-1}\left(\mathrm{r}^{2}=0.8691\right)$ for STM 1 and $10^{4} \mathrm{CFU} \mathrm{mL} \mathrm{L}^{-1}\left(\mathrm{r}^{2}=0.8529\right)$ for SE 1 . As expected, the parental probes have shown a poor linearity and low LOD values for both, STM $110^{7}$ CFU mL ${ }^{-1}\left(r^{2}=0.6535\right)$ and SE $110^{9} \mathrm{CFU} \mathrm{mL}^{-1}\left(r^{2}=0.5151\right)$, respectively. These results confirm Sal-3 and Sal-5 probes as the most optimal substrates for detecting Salmonella in a sensitive manner, and with the capability for detecting bacteria in less than $8 \mathrm{~h}$.

\subsection{Performance of the nuclease assays in BPW-MLN field samples}

To prove the utility of the two candidate-probes to detect Salmonella in field samples, we designed a blind study carried out on 15 Salmonella-positive and 15 Salmonella-negative BPW-MLN samples 
previously analysed by the ISO 6579 (Table S3) and PCR-InvA methods (Figure S3). Figure 6 shows the results obtained by the three different techniques. As shown in the growth curves assay, Sal-3 and Sal- 5 candidate-probes performed better than the parental-probe in all cases under optimal conditions. Notably, $100 \%$ correlation was observed between Sal-3 and Sal-5 Salmonella nuclease activity method in BPW-MLN field cultures and both the microbiological gold standard and molecular methodologies. These results underline the potential of the Salmonella probes described herein and their applicability to real samples analysis.

\section{DISCUSSION}

Detection of pathogenic bacteria in food products is a critical parameter to determine quality control and food safety. The current detection methods are laborious and time consuming, with procedures mostly based on bacterial cultures [26, 27]. Several microbiology techniques, immunoassays, PCR and the standard methodology for detecting Salmonella (ISO 6579:2002/Amd 1:2007) have been described, however they require enriched culture samples of at least 24h [26-30]. Other technologies such as sequencing and mass spectroscopy have shown some promising detection capabilities [31], however, with a time to diagnosis of more than a day, remaining a significant drawback. More specifically, Salmonella detection in food samples requires days to weeks until confirmation. In contrast, the worldwide demand for food is in a continuous growth to fulfil global requirements. Thus, novel and faster methodologies are needed to identify Salmonella and other pathogens, with detection times catching up with the velocity of the food supply chain, "from farm to fork". Herein, we report on a novel detection system based on oligonucleotide probes with the capability to detect Salmonella in less than 8 hours. This strategy uses the nuclease activity derived from Salmonella as biomarker. Our results have shown the screening and identification of two oligonucleotide sequences (substrates) as the most promising probes for detecting Salmonella. Moreover, we demonstrated the potential of these selected oligonucleotide probes to detect Salmonella cultures in a sensitive and specific manner. These probes were able to recognize nuclease activity derived from $10 \mathrm{CFU} \cdot \mathrm{mL}^{-1}$ of bacteria after a short sub-culture between 6 and 8 hours. In addition, these probes have the capability to differentiate Salmonella from other non-specific bacteria that are commonly found as food contaminants. To complete the characterization of our Salmonella probes, we performed a blind study using lymph nodes, which are a well-standardized sample for Salmonella testing in pork meat. Our oligonucleotide probe system has reported $100 \%$ correlation with two other independent entities, confirming the great potential of this technology to be implemented as an alternative method for detecting Salmonella in food products. Notably, this study demonstrates that significant reduction in detection time for targeting some specific bacteria is possible, and technologies such as nucleic acid probes could help to adapt/synchronize the food safety methods with the food supply chain.

Our approach still needs a short bacterial culture to get an accurate detection of the pathogen, which could pose limitations for certain applications where the means for growing bacteria is difficult. Thus, we envision that additional reduction in detection time could be achieved by exploring other signal transduction mechanisms for the nuclease activity readout, optimizing culture media and further 
exploration of our probe system, allowing Salmonella detection in 1-4 hours. These future improvements could facilitate the integration of our Salmonella probes into portable devices. Therefore, the complete elimination or minimal need for bacterial cultures remains an attractive challenge for reducing detection times.

\section{AUTHORS CONTRIBUTION}

I.M. Performed and analyzed nuclease activity experiments for the screening, optimization and validation of the Salmonella probes. Oversaw general aspects of the study and helped to write the manuscript. V.G. Prepared all microbiology cultures and the field samples used in this study, performed the PCR experiments, CFU counting, and helped to write the manuscript. LIH. Analysed and plotted all the nuclease activity data, helped to design the Salmonella probes and wrote de manuscript. JB. Performed and analysed the nuclease activity experiments for the Salmonella serotypes. NB. Performed and analysed the nuclease activity experiments for the initial screening. BSR. Helped with the development of the experimental validation and culture preparations. MJG. Developed the microbiology validation strategy of this study, oversaw the execution of the microbiology experiments and helped to write the manuscript. FJH. Developed the conceptual strategy for the screening and selection of the Salmonella probes, analysed the nuclease activity data, designed the optimal Salmonella probes, and wrote the manuscript. All the authors revised the manuscript.

\section{CONFLICT OF INTEREST}

$\mathrm{LIH}$ and FJH are inventors in 2 patents that describe the use of nucleic acid probes for detecting bacteria. SOMAprobes is a company that develops nucleic acid probes similar to those reported in this study for Salmonella, food and clinical applications. MJG and VG are inventors of the patent number P201830206 entitled "Nucleic acid probes and their use as a substrate in a method for the detection of Salmonella".

\section{ACKNOWLEDGEMENTS}

This work was supported by MINNECO [Grant: PTQ-16-08414] (IM), The Centre for the Development of Industrial Technology [CDTI Grant: 20161256] (SOMAprobes), Departamento de Industria, Energia e Innovación of the Navarra government, Spain [Grant: 2016 PT071 and 2017 PT031], Swedish Government Strategic Research Area in Materials Science on Advanced Functional Materials at Linköping University (Faculty Grant SFO-Mat-LiU No. 2009-00971) for financial support and The Knut and Alice Wallenberg foundation is acknowledged for generous support $(\mathrm{FJH})$. 


\section{REFERENCES}

1. Pires, S.M., L. de Knegt, and T. Hald, Estimation of the relative contribution of different food and animal sources to human Salmonella infections in the European Union, 2011, National Food Institute Technical University of Denmark: Parma, Italy. p. 80.

2. Shah, D.H., et al., Cell invasion of poultry-associated Salmonella enterica serovar Enteritidis isolates is associated with pathogenicity, motility and proteins secreted by the type III secretion system. Microbiology, 2011. 157(5): p. 1428-45.

3. Cellucci, T., et al., A 10-year retrospective review of Salmonella infections at the Children's Hospital in London, Ontario. Can J Infect Dis Med Microbiol, 2010. 21(2): p. 78-82.

4. Su, L.H. and C.H. Chiu, Salmonella: clinical importance and evolution of nomenclature. Chang Gung Med J, 2007. 30(3): p. 210-9.

5. Mughini-Gras, L., Enserink, R., Friesema, I., Heck, M., van Duynhoven, Y. and van Pelt, W. , Risk Factors for Human Salmonellosis Originating from Pigs, Cattle, Broiler Chickens and Egg Laying Hens: A Combined Case-Control and Source Attribution Analysis. Plos One, 2014. 9(2): p. 1-9.

6. Commission, E., The European Union summary report on trends and sources of zoonoses, zoonotic agents and food-borne outbreaks in 2015, 2016, European Food Safety Authority and European Centre for Disease Prevention and Control: EFSA Journal. p. 231.

7. CDC, Foodborne Diseases Active Surveillance Network FoodNet 2015 Surveillance Report (Final Data), 2017, Department of Health and Human Services, CDC: Atlanta, Georgia, U. S.

8. Scallan, E., et al., Foodborne Illness Acquired in the United States - Major Pathogens. CDC. Emerging Infectious Diseases, 2011. 17(1): p. 9.

9. Odumeru, J.A., León-Velarde, C. G., Salmonella Detection Methods for Food and Food Ingredients, in Salmonella - A Dangerous Foodborne Pathogen, B.S.M. Mahmoud, Editor. 2012, InTech: University of Guelph, Guelph, Ontario, Canada. p. 373 - 392.

10. Zadernowska, A., Chajecka, W., Detection of Salmonella spp. Presence in Food in Salmonella - A Dangerous Foodborne Pathogen, B.S.M. Mahmoud, Editor. 2012, InTech: University of Warmia and Mazury in Olsztyn, Faculty of Food Sciences Chair of Industrial and Food Microbiology, Poland. p. 393 412.

11. Almeida, C., et al., Detection of Salmonella enterica serovar Enteritidis using real time PCR, immunocapture assay, PNA FISH and standard culture methods in different types of food samples. Int $\mathrm{J}$ Food Microbiol, 2013. 161(1): p. 16-22.

12. Ahmed, O.B., Asghar, A. H., El-Rahim, I. H. A. A., Al, H., Detection of Salmonella in Food Samples by Culture and Polymerase Chain Reaction Methods. Journal of Bacteriology \& Parasitology, 2014. 5(3): p. $187-189$.

13. Law, J.W., et al., Rapid methods for the detection of foodborne bacterial pathogens: principles, applications, advantages and limitations. Front Microbiol, 2014. 5: p. 770.

14. Mainar-Jaime, R.C., et al., Sensitivity of the ISO 6579:2002/Amd 1:2007 standard method for detection of Salmonella spp. on mesenteric lymph nodes from slaughter pigs. J Clin Microbiol, 2013. 51(1): p. 89-94.

15. Hernandez, F.J., et al., Noninvasive imaging of Staphylococcus aureus infections with a nucleaseactivated probe. Nat Med, 2014. 20(3): p. 301-6.

16. V. Garrido, S.S., B. San Román, A. Zabalza-Baranguá, Y. Díaz-Tendero, C. de Frutos, R. C. MainarJaime and M. J. Grilló, Simultaneous infections by different Salmonella strains in mesenteric lymph nodes of finishing pigs. BMC Veterinary Research, 2014. 10(59): p. 1-6.

17. B. San Román, V.G., S. Sanchéz, I. Martinez-Ballesteros, J. Garaizar, R. C. Mainar-Jaime, L. MiguraGarcia and M. J. Grilló, Relationship between Salmonella infection, shedding and serology in fattening pigs in low-moderate prevalence areas. Zoonoses Public Health, 2017. 65(5): p. 1-9.

18. ISO, ISO 6579:2002/Amd 1:2007. Detection of Salmonella spp. in animal faeces and in environmental samples from the primary production stage, amendment 1, annex D. In Microbiology of food and animal feeding stuffs. Horizontal method for the detection of Salmonella spp., 2007, International Organization Standardization: Geneva, Switzerland.

19. Arnold, T., et al., Impact of invA-PCR and Culture Detection Methods on Occurrence and Survival of Salmonella in the Flesh, Internal Organs and Lymphoid Tissues of Experimentally Infected Pigs. Journal of Veterinary Medicine B, 2004. 51: p. 459-463.

20. Shrivastava, A. and V.B. Gupta, Methods for the determination of limit of detection and limit of quantitation of the analytical methods. Chronicles of Young Scientists (actual Drug Development and Therapeutics), 2011. 2(1): p. 21-25.

21. Gray, P.M., M.S. Rhee, and D.H. Kang, The correlation method for rapid monitoring of Escherichia coli in foods. Letters in Applied Microbiology, 2002. 34: p. 269-273.

22. Ki Suk, J., G.E. Ji, and I.K. Hwang, Assay of b-Glucosidase Activity of Bifidobacteria and the Hydrolysis of Isoflavone Glycosides by Bifidobacterium sp. Int-57 in Soymilk Fermentation. Journal of Microbiology and Biotechnology, 2002. 12(1): p. 8-13. 
23. Siragusa, G.R., D.H. Kang, and C.N. Cutter, Monitoring the microbial contamination of beef carcass tissue with a rapid chromogenic Limulus amoebocyte lysate endpoint assay. Letters in Applied Microbiology, 2000. 31: p. 178-183.

24. Flenker, K.S., et al., Rapid Detection of Urinary Tract Infections via Bacterial Nuclease Activity. Molecular Therapy, 2017. 25(6): p. 1353-1362.

25. Xu, L., et al., In-field detection of multiple pathogenic bacteria in food products using a portable fluorescent biosensing system. Food Control, 2016. 75: p. 21-28.

26. Priyanka, B., R.K. Patil, and S. Dwarakanath, A review on detection methods used for foodborne pathogens. Indian Journal of Medical Research, 2016. 144(3): p. 327-338.

27. Ricke, S.C., et al., Molecular-based identification and detection of Salmonella in food production systems: current perspectives. Journal of Applied Microbiology, 2018. 125: p. 313-327.

28. Radhika, M., et al., A novel multiplex PCR for the simultaneous detection of Salmonella enterica and Shigella species. Brazilian Journal of Microbiology, 2014. 45(2): p. 667-676.

29. Sharma, V.K. and C.S. A., Simultaneous Detection of Salmonella Strains and Escherichia coli O157:H7 with Fluorogenic PCR and Single-Enrichment-Broth Culture. Applied and Environmental Microbiology, 2000. 66(12): p. 5472-5476.

30. Techathuvanan, C., F.A. Draughon, and D.H. D'Souza, Real-time reverse transcriptase PCR for the rapid and sensitive detection of Salmonella typhimurium from pork. Journal of Food Protection, 2010. 73(3): $p$. 507-514.

31. Bell, R. L., Jarvis, K. G., Ottesen, A. R., McFarland, M. A., Brown, E. W, Recent and emerging innovations in Salmonella detection: a food and environmental perspective, Microbial biotechnology, 2016. 9(3), 279292. 


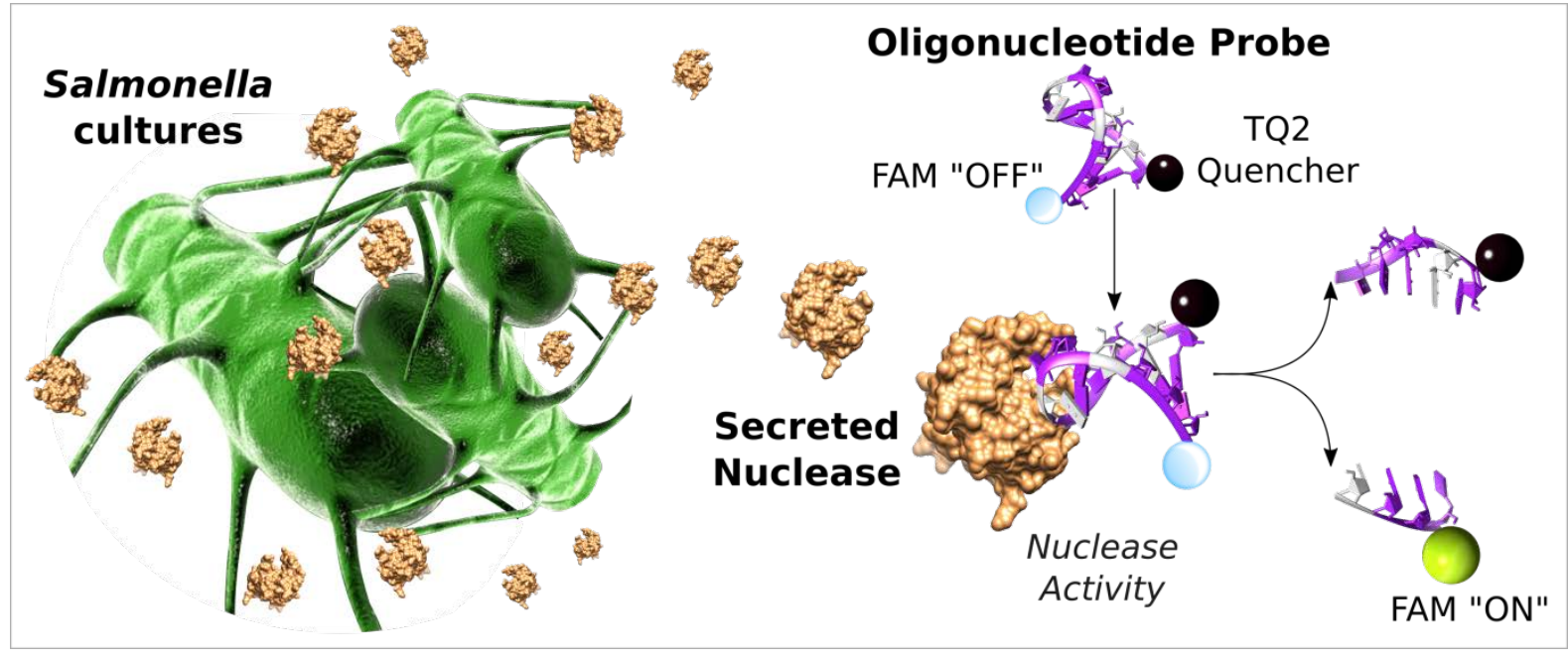

Figure 1. Schematic concept for targeting nuclease activity derived from Salmonella by oligonucleotide probes. 


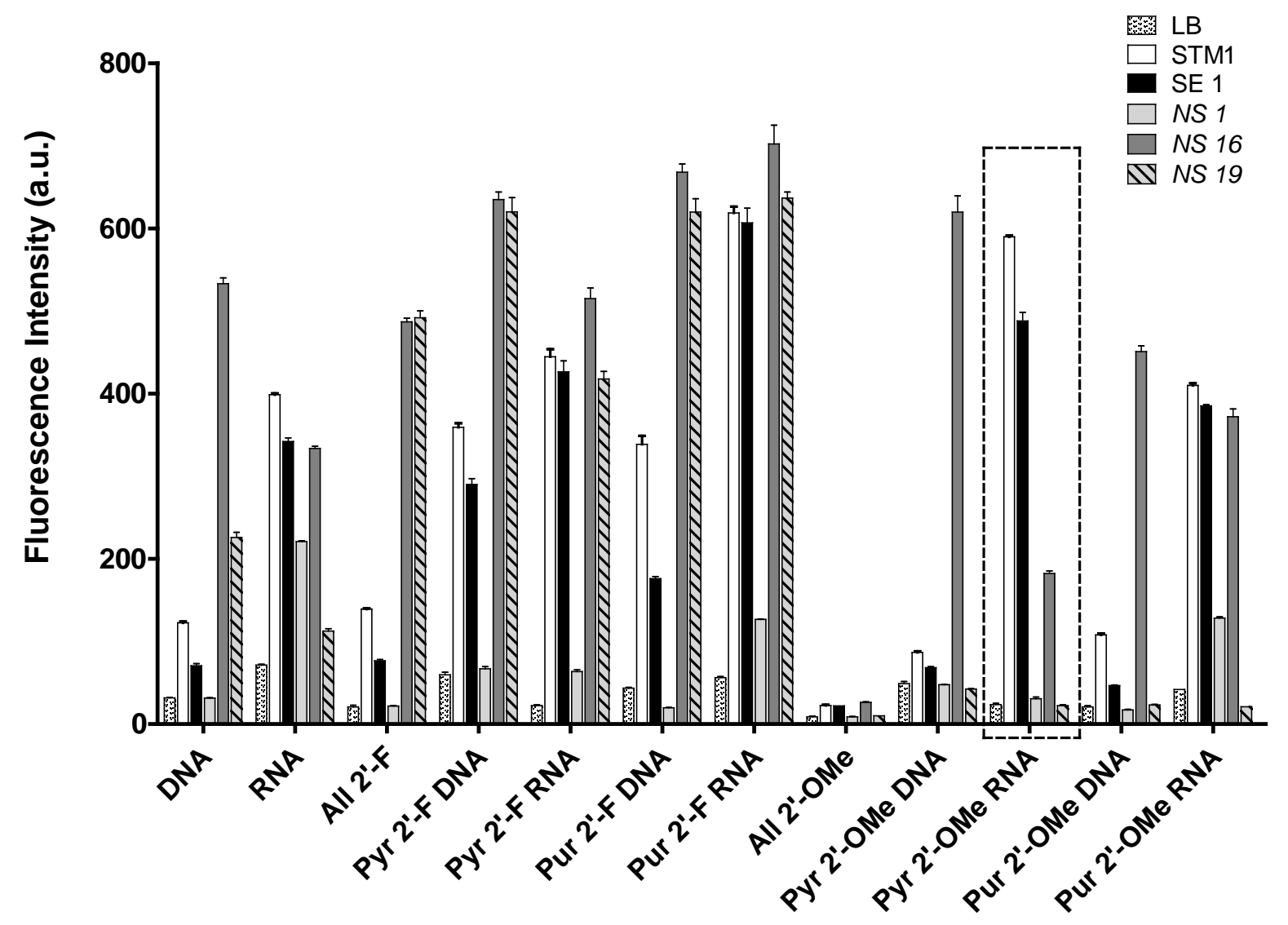

Figure 2. Screening of the Salmonella derived nuclease activity. Global nuclease activity derived from Salmonella is reported as increase in the mean fluorescence intensity as result of degradation of each probe. The bars represent the average of triplicate fluorescence measurements $( \pm s . d)$. Data are representative of at least 3 individual experiments. The dashed line rectangle emphasizes the Pyr-2'OMe-RNA probe (Parental), the one that shown the best ability for discriminating Salmonella from the control bacterial cultures. 


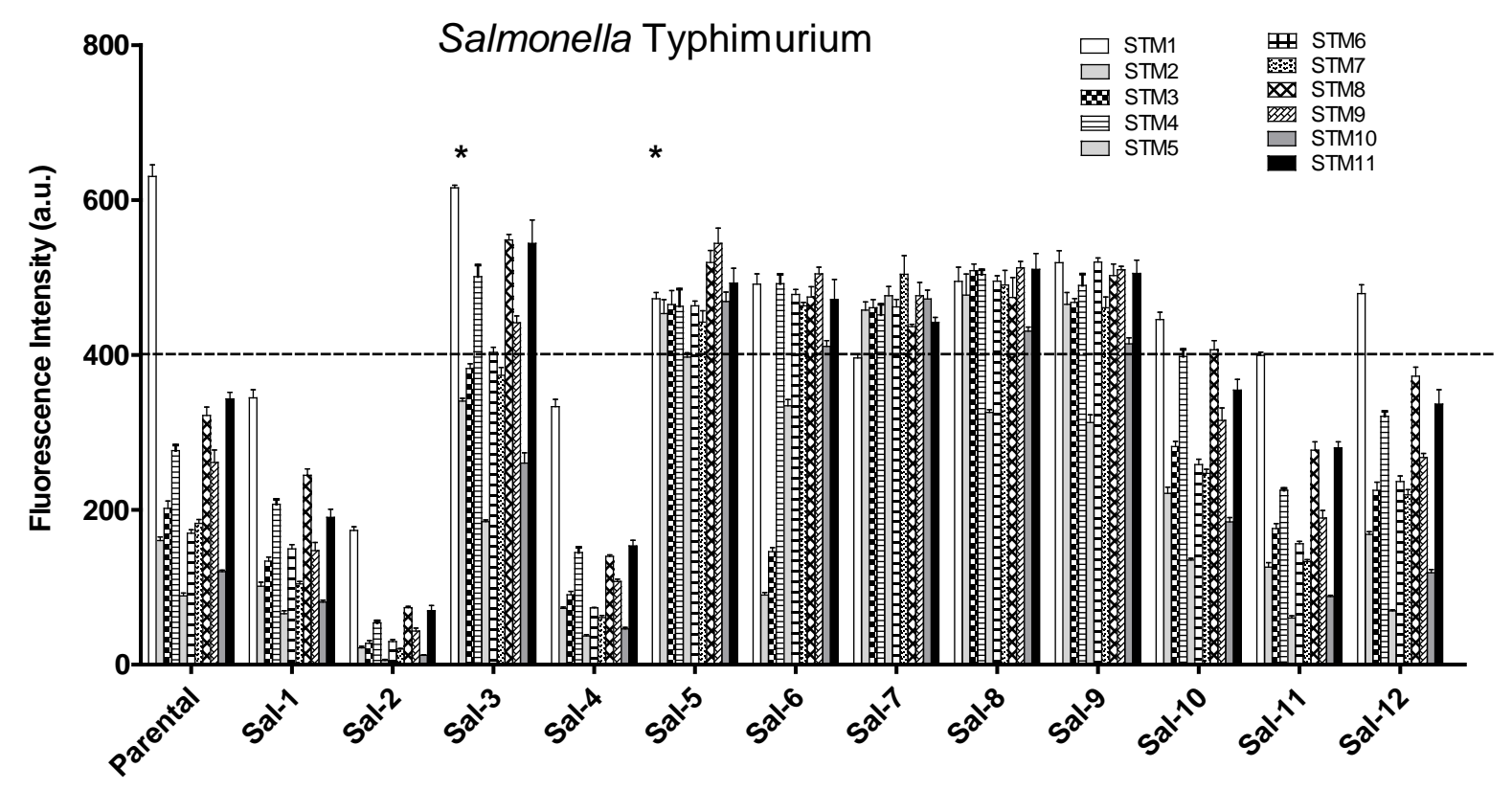

Figure 3a. Nuclease activity profile of 11 cultures of Salmonella Typhimurium using the Parental probe and 12 derivative Salmonella probes. The bars represent the mean of triplicate fluorescence measurements ( \pm s.d.). Data are representative of at least 3 individual experiments. The asterisks indicate the most promising candidate probes determined for targeting Salmonella. The dashed line is an arbitrary upper threshold to guide the eye and facilitate the results interpretation. 


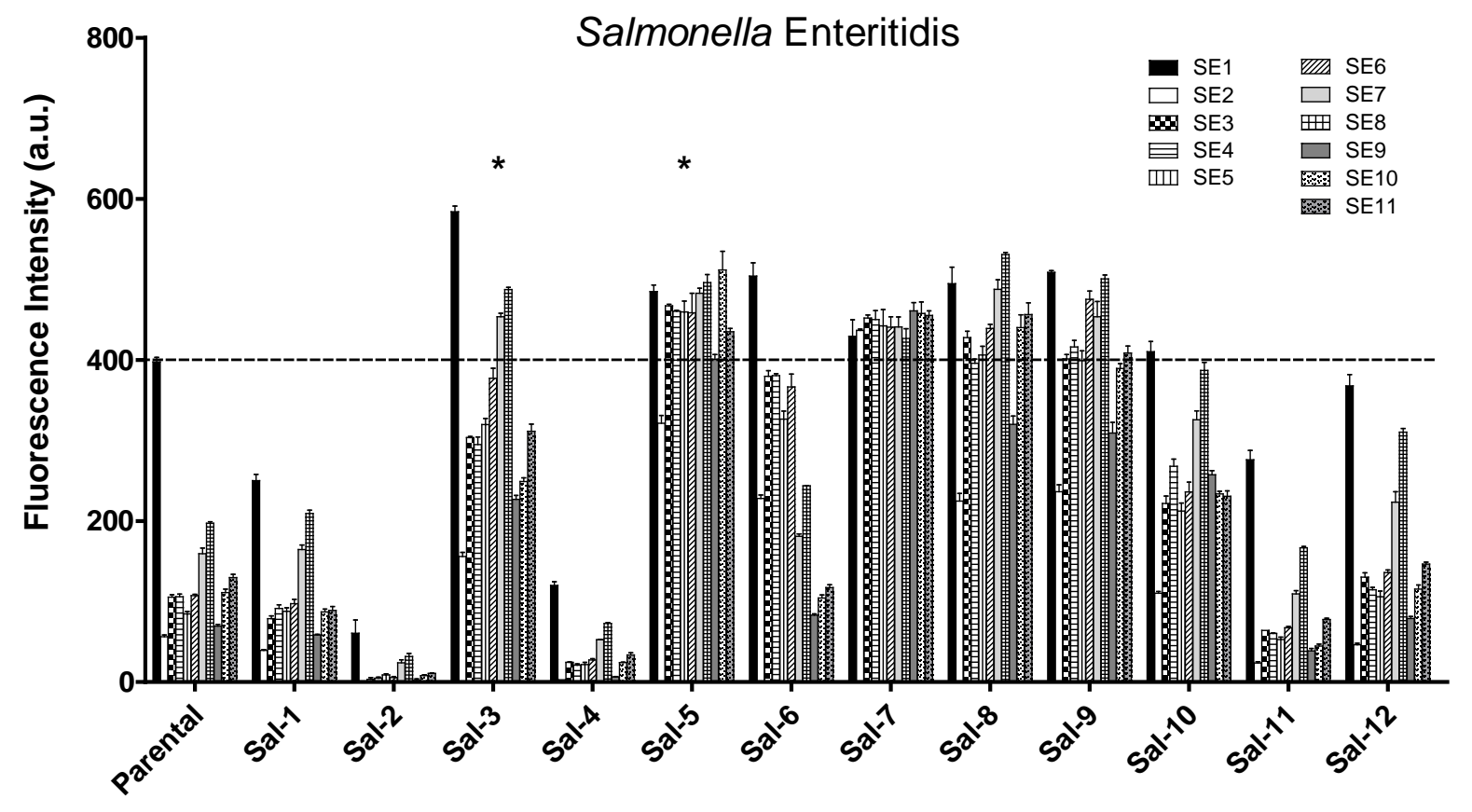

Figure 3b. Nuclease activity profile of 11 cultures of Salmonella Enteritidis using the Parental probe and 12 derivative Salmonella probes. The bars represent the mean of triplicate fluorescence measurements ( \pm s.d.). Data are representative of at least 3 individual experiments. The asterisks indicate the most promising candidate probes determined for targeting Salmonella. The dashed line is an arbitrary upper threshold to guide the eye and facilitate the results interpretation. 


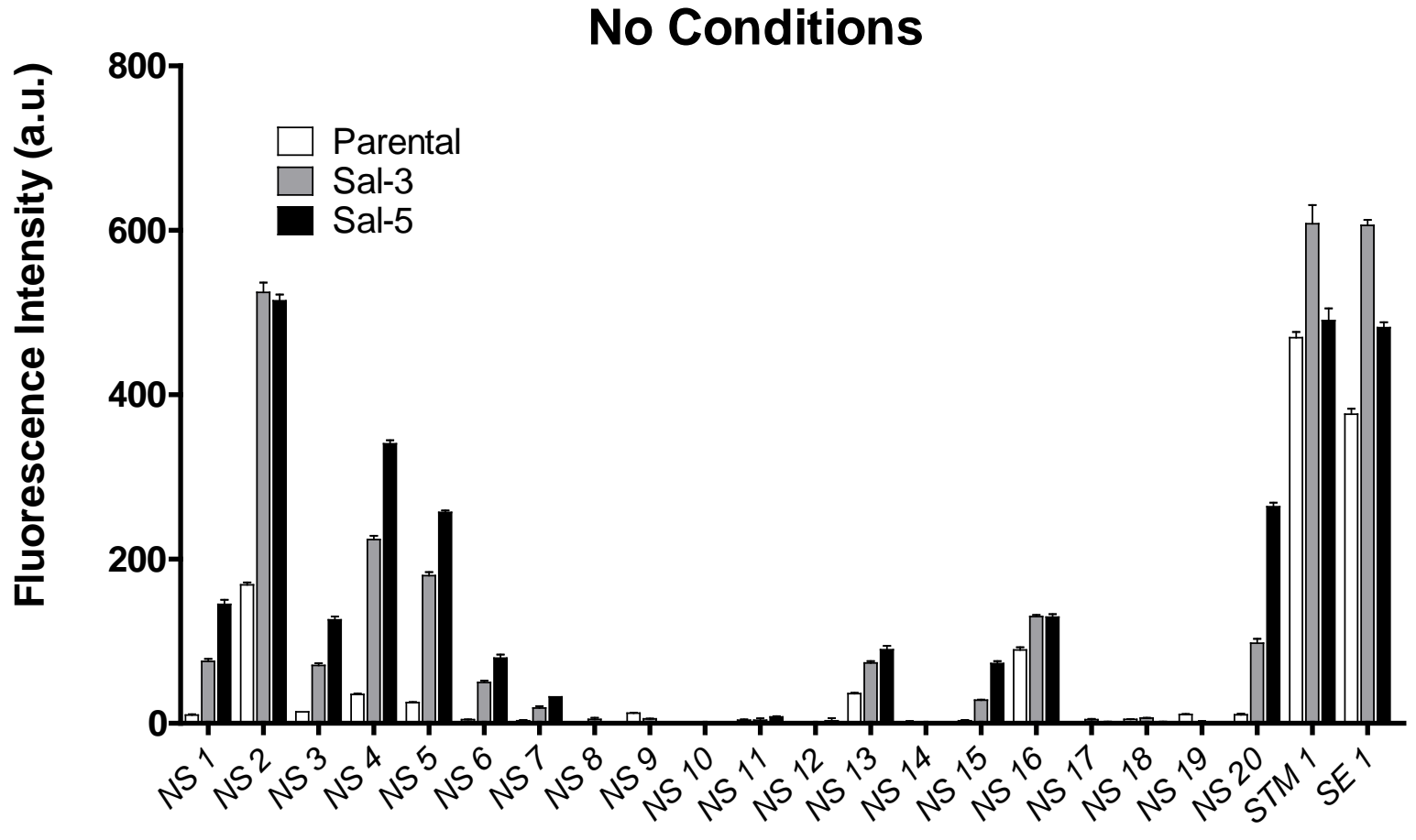

Figure 4a. Specificity study of the Salmonella probes under standard conditions. Various bacterial cultures were tested using Parental, Sal- 3 and Sal-5 probes under standard conditions $-1 \mathrm{~h}$ incubation time at $37^{\circ} \mathrm{C}$ and $\mathrm{PBS}+/+-$ for the nuclease activity assay. 


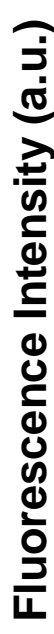

With Conditions

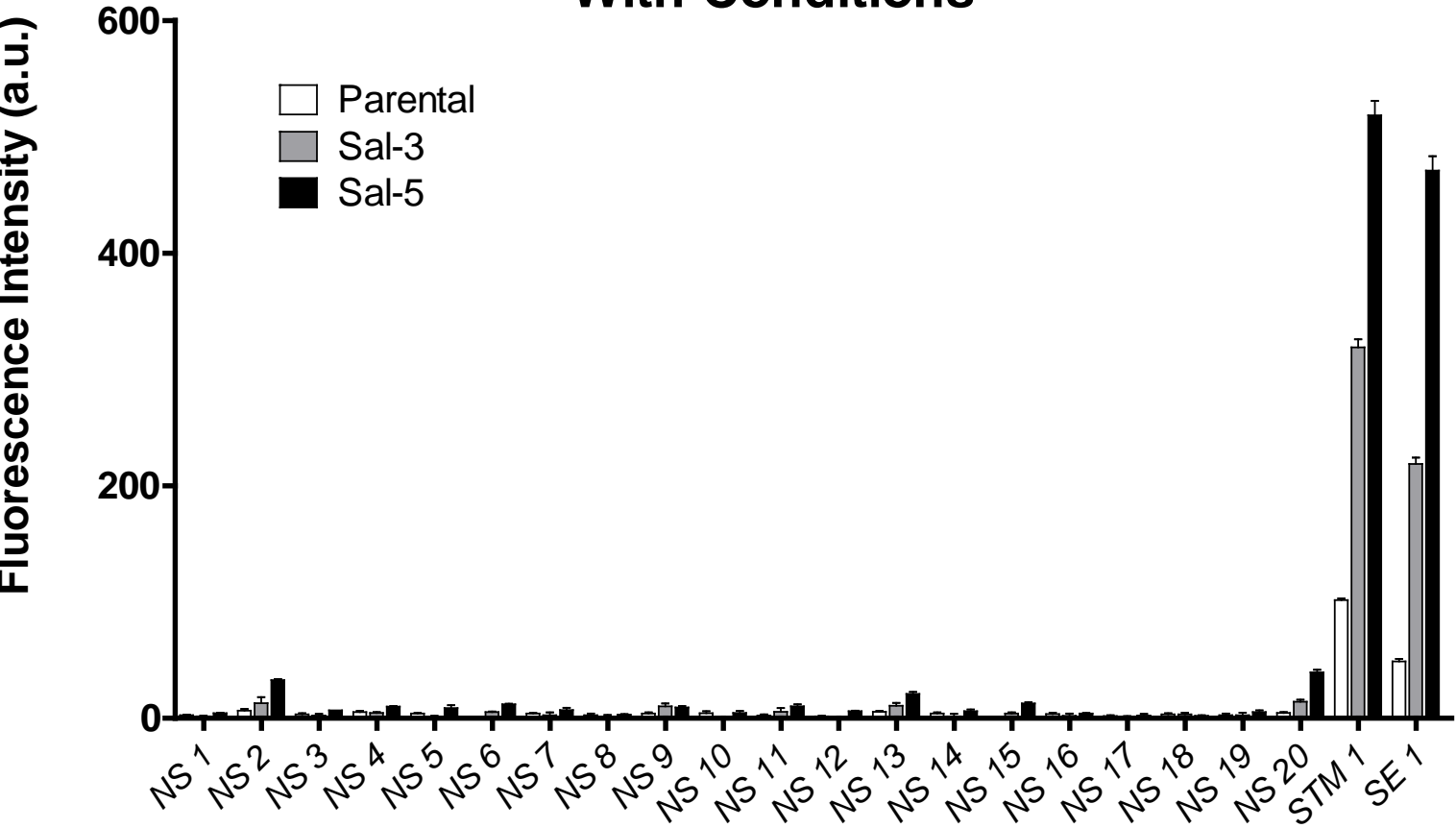

Figure 4b. Specificity study of the Salmonella probes under optimized conditions. Various bacterial cultures were tested using Parental, Sal-3 and Sal-5 probes under optimized conditions - 15 min. incubation time at $37^{\circ} \mathrm{C}$ and addition of EGTA chelating agent - for the nuclease activity assay. 


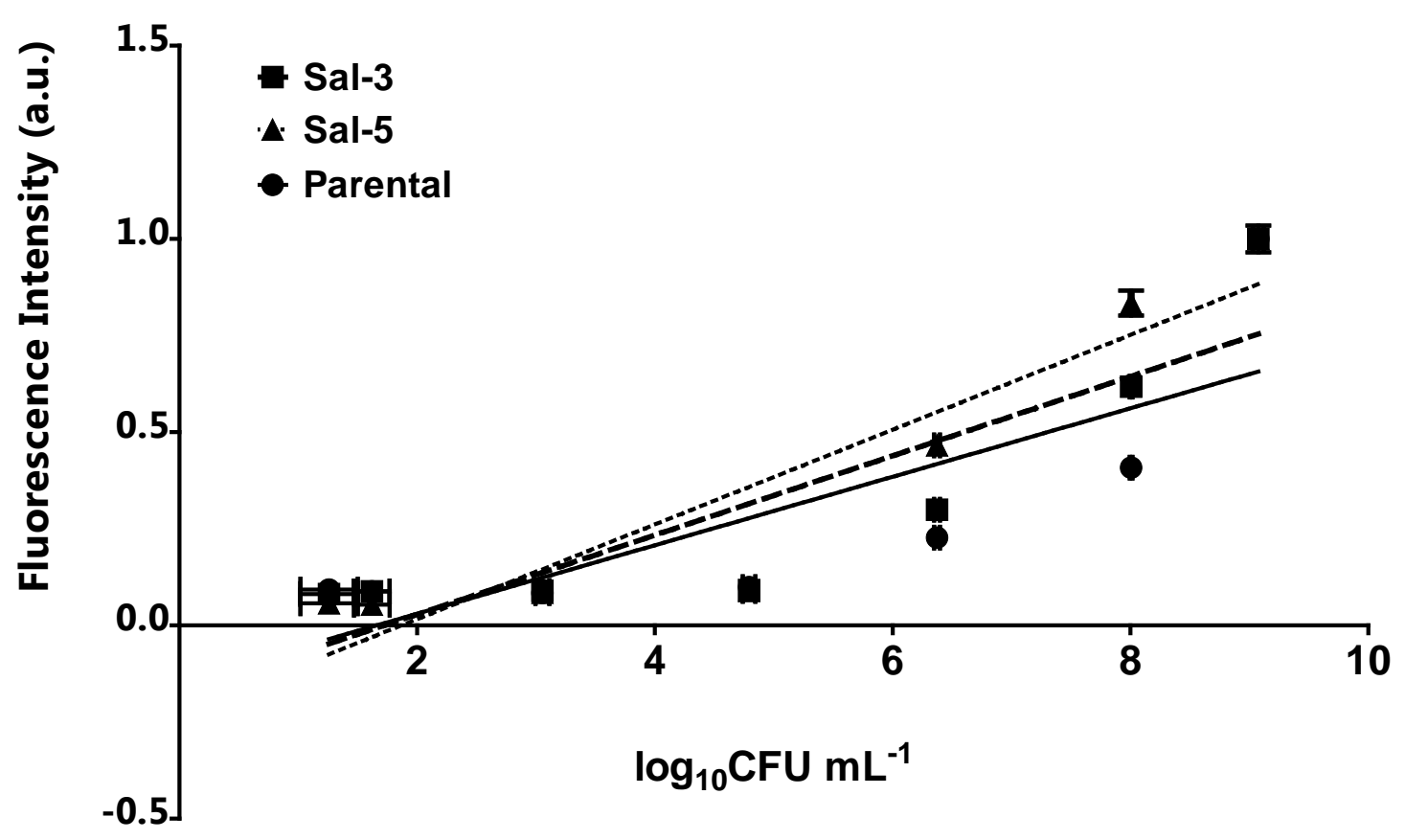

Figure 5a. Determination of the LOD Salmonella Typhimurium (STM 1). Correlation between normalized fluorescence and bacteria concentration of the cultures of STM 1 using the probes Sal-3, Sal-5 and Parental. The long dashed, dashed and solid lines correspond to the linear regression determined to calculate the LOD with each probe, respectively. 


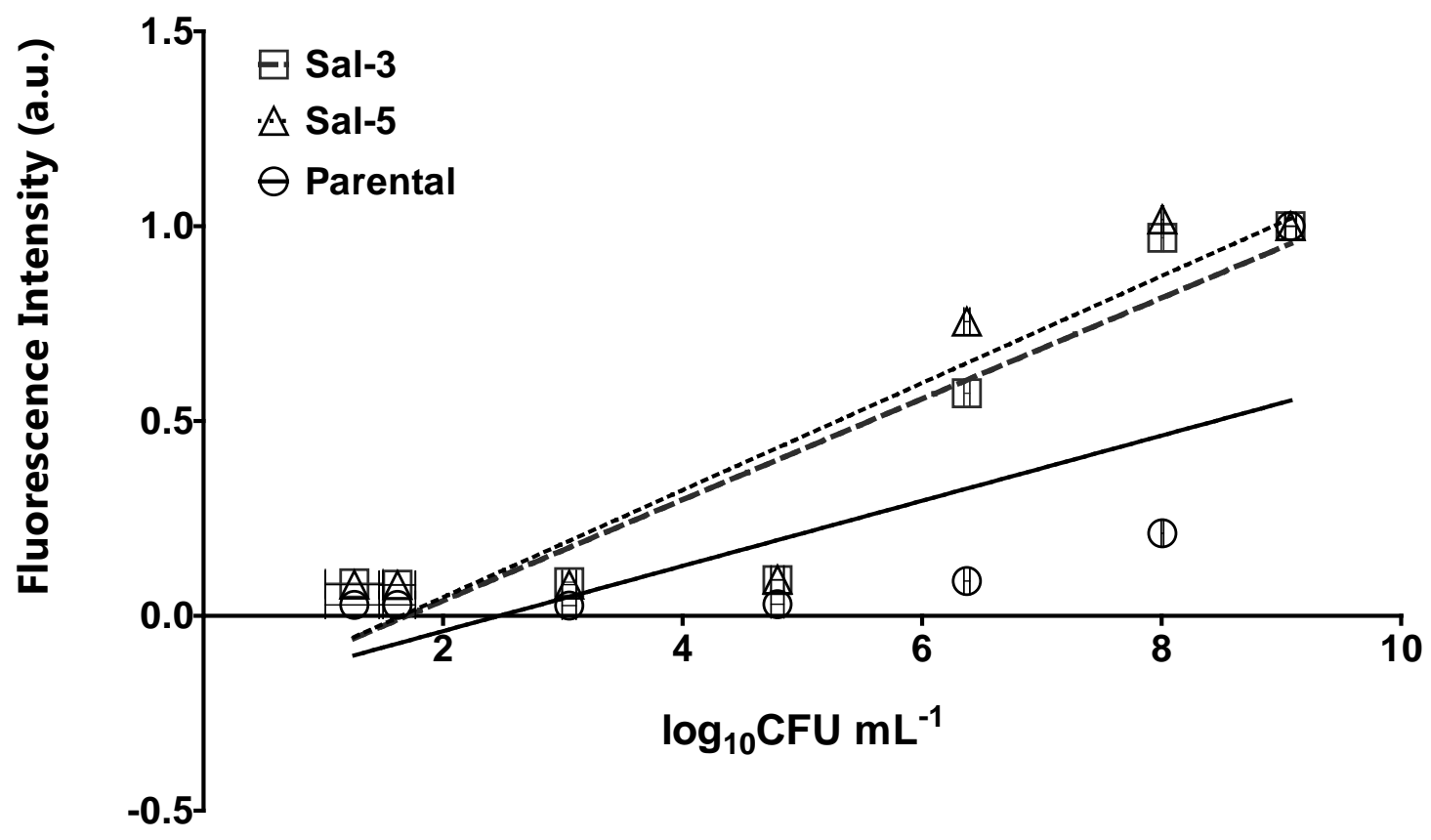

Figure 5b. Determination of the LOD for Salmonella Enteritidis (SE 1). Correlation between normalized fluorescence and bacteria concentration of SE 1 cultures using the probes Sal-3, Sal-5 and Parental. The long dashed, dashed and solid lines correspond to the linear regression determined to calculate the LOD with each probe, respectively. 


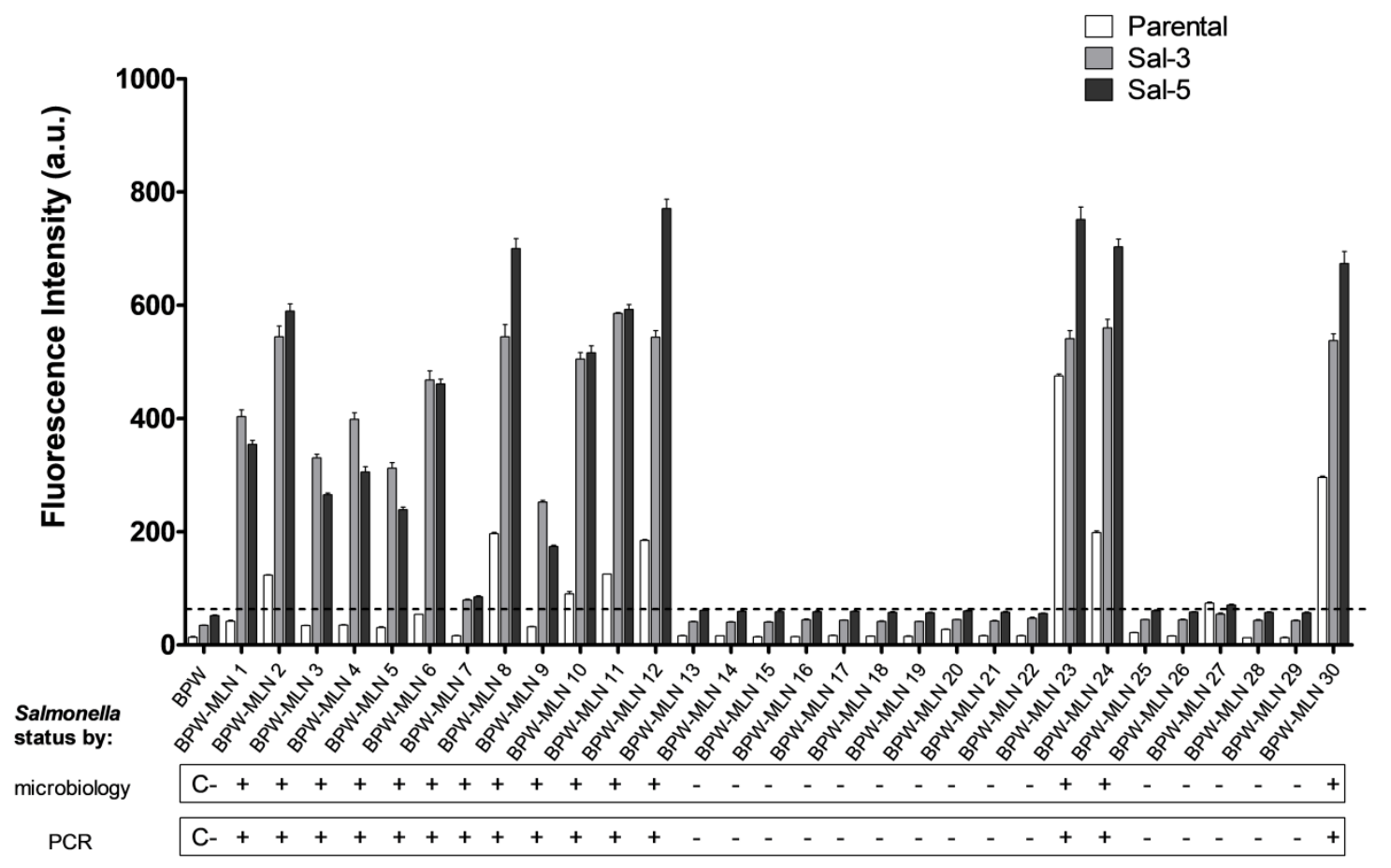

Figure 6. Blind experiment to determine the presence or absence of Salmonella in pig lymph nodes. Bars represent the specific nuclease activity derived from the lymph nodes samples using Parental (white bars), Sal-3 (grey bars) and Sal-5 (black bars) oligonunucleotide probes. BPW buffer was used as negative control for these samples. Correlation of $100 \%$ was observed between the standard methods and Salmonella probes. The dashed line is an arbitrary threshold to guide the eye and diferentiate the negative (bars below the line) from the positive samples (bars above the line, including BPW-MLN7 sample). 


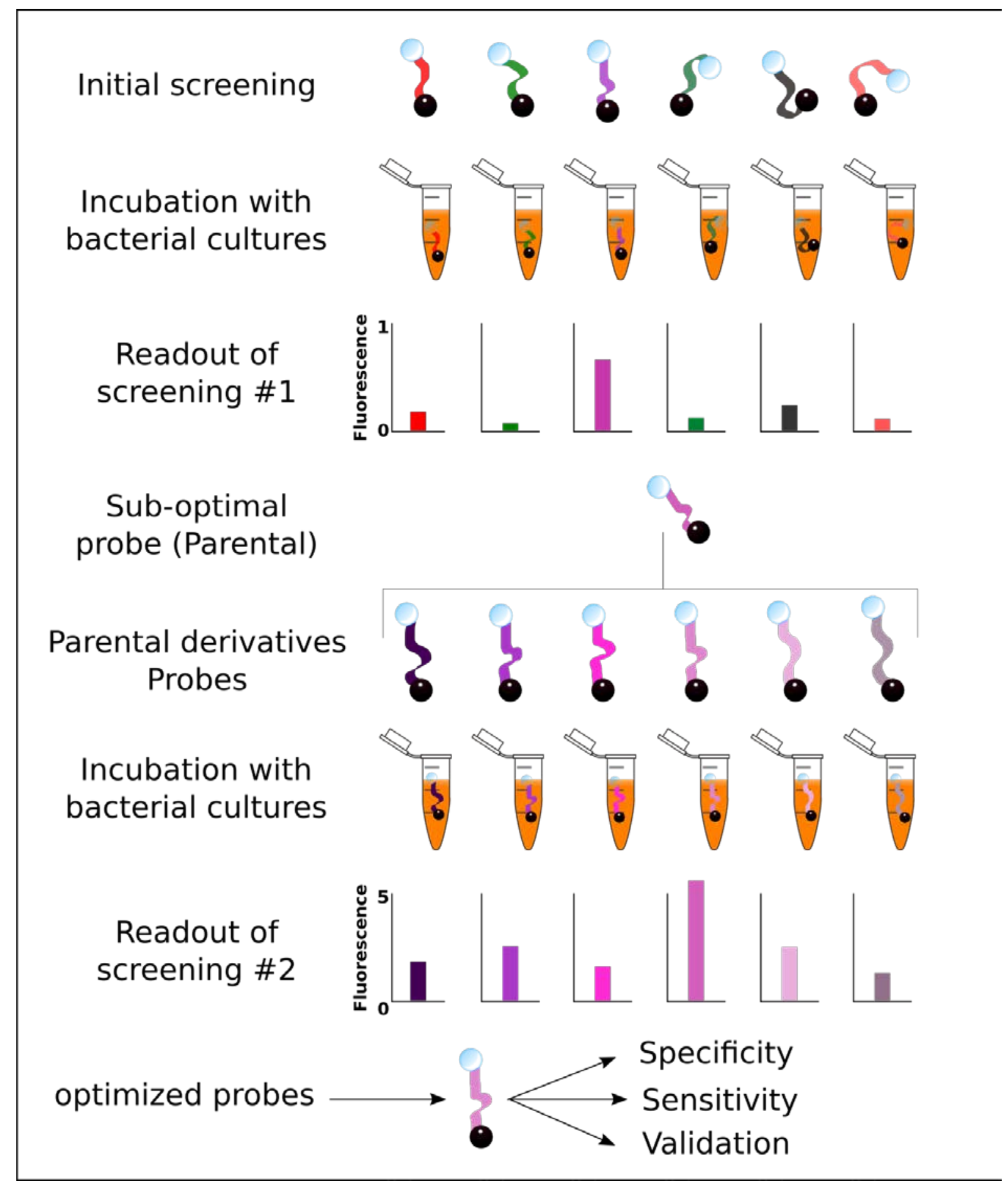

Figure S1. Schematic process of the screening for the optimized oligonucleotide probes. 
Table S1: List of strains and BPW-MLN samples used in this work.

\begin{tabular}{|c|c|}
\hline Code & Characteristics \\
\hline Salmonella strains: & Field isolates from fattening pigs: \\
\hline STM1 & S. Typhimurium ATCC 14028 \\
\hline STM2 to STM11 & S. Typhimurium from different animals \\
\hline SE1 to SE11 & S. Enteritidis from different animals \\
\hline $\mathrm{S} 1$ & S. Typhimurium monophasic \\
\hline $\mathrm{S} 2$ & S. Abaetetuba \\
\hline S3 & S. Agona \\
\hline S4 & S. Amsterdam \\
\hline S5 & S. Anatum \\
\hline S6 & S. enterica subesp. arizonae \\
\hline S7 & S. Bardo \\
\hline S8 & S. Bovismobificans \\
\hline S9 & S. Braenderup \\
\hline S10 & S. Brandenburg \\
\hline S11 & S. Bredeney \\
\hline S12 & S. Coeln \\
\hline S13 & S. Derby \\
\hline S14 & S. enterica subesp. diarizonae \\
\hline S15 & S. Enteritidis \\
\hline S16 & S. Gaminara \\
\hline S17 & S. Give \\
\hline S18 & S. Goldcoast \\
\hline S19 & S. Hadar \\
\hline S20 & S. Havana \\
\hline S21 & S. Infantis \\
\hline S22 & S. Indiana \\
\hline S23 & S. Kapemba \\
\hline S24 & S. Kedogou \\
\hline $\mathrm{S} 25$ & S. Kottbus \\
\hline S26 & S. Lexington \\
\hline S27 & S. Livingstone \\
\hline S28 & S. London \\
\hline S29 & S. Meleagridis \\
\hline S30 & S. Mikawasima \\
\hline S31 & S. Mishmarhaek \\
\hline S32 & S. Montevideo \\
\hline S33 & S. Muenchen \\
\hline S34 & S. Newport \\
\hline S35 & S. Nottingham \\
\hline S36 & S. Ohio isolated \\
\hline S37 & S. Oranienburg \\
\hline S38 & S. Poona \\
\hline S39 & S. Reading \\
\hline $\mathrm{S} 40$ & S. Rissen \\
\hline S41 & S. enterica subesp. salamae \\
\hline
\end{tabular}




\begin{tabular}{ll}
\hline S42 & S. Szentes \\
S43 & S. Tennessee \\
S44 & S. Thompson \\
S45 & S. Toulon \\
S46 & S. Umbilo \\
S47 & S. Urbana \\
S48 & S. Wien \\
S49 & $4,12:$ i:- \\
S50 & 6,7:-:1,5 \\
S51 & $9,12:-:-$ \\
& \\
\hline Non-Salmonella (NS) & Field isolates from fattening pigs: \\
strains: & Escherichia coli ATCC 25922 and other 4 strains \\
NS1 to NS5 & isolated from food samples and fattening pigs \\
& Enterococcus faecalis isolated from fattening pigs \\
NS6 & Hafnia alvei isolated from pig environment \\
NS7 and NS8 & Moellerella winsconsiensis isolated from food samples \\
NS9 & Oceanobacillus oncorhynchi isolated from food \\
NS10 & samples \\
& Pseudomonas aeruginosa isolated from fattening pigs \\
NS11 and NS12 & Proteus mirabilis isolated from fattening pigs \\
NS13 & Providencia rustigianii isolated from food samples \\
NS14 & Proteus vulgaris isolated from fattening pigs \\
NS15 & Staphylococcus aureus isolated from food samples \\
NS16 and NS17 & Serratia liquefaciens isolated from pig environment \\
NS18 & Streptococcus pyogenes isolated from food samples \\
NS19 & Klebsiella pneumoniae isolated from food samples \\
NS20 & \\
& \\
&
\end{tabular}


Table S2. Oligonucleotide probes names and respective DNA or RNA sequences.

Note: Uppercase red T, A, C, G = DNA

Lowercase blue $\mathrm{u}, \mathrm{a}, \mathrm{c}, \mathrm{g}=\mathrm{RNA}$

$\mathrm{m}=$ 2'-O-Methyl ribose modification

$\mathrm{f}=2^{\prime}$ '- Fluor ribose modification

\begin{tabular}{|l|l|}
\hline \multicolumn{1}{|c|}{ Seq. Name } & \multicolumn{1}{c|}{ Sequence } \\
\hline P1_DNA & FAM // TCTCGTACGTTC // TQ2 \\
\hline P2_RNA & FAM // ucucguacguuc // TQ2 \\
\hline P3_All 2'-F & FAM // fUfCfUfCfGfUfAfCfGfUfUfC // TQ2 \\
\hline P4_Pyr 2'-F DNA & FAM // fTfCfTfCgfTafCgfTfTfC // TQ2 \\
\hline P5_Pyr 2'-F RNA & FAM // fUfCfUfCgfUafCgfUfUfC // TQ2 \\
\hline P6_Pur 2'-F DNA & FAM // TfAfACfGTfACfGfGTC // TQ2 \\
\hline P7_Pur 2'-F RNA & FAM // ufAfAcfGufAcfGfGuc // TQ2 \\
\hline P8_All 2'-OMe & $\begin{array}{l}\text { FAM // mUmCmUmCmGmUmAmCmGmUmUmC } \\
\text { // TQ2 }\end{array}$ \\
\hline P9_Pyr 2'-OMe DNA & FAM // mTmCmTmCgmTamCgmTmTmC // TQ2 \\
\hline P10_Pyr 2'-OMe RNA & FAM // mUmCmUmCgmUamCgmUmUmC // TQ2 \\
\hline P11_Pur 2'-OMe DNA & FAM // TmAmACmGTmACmGmGTC // TQ2 \\
\hline P12_Pur 2'-OMe RNA & FAM // umAmAcmGumAcmGmGuc // TQ2 \\
\hline Sal 1_Pyr 2'-OMe RNA & FAM // mUmCmUmCmCmUamCmUmUmUmC // \\
\hline Sal 2_Pyr 2'-OMe RNA
\end{tabular}




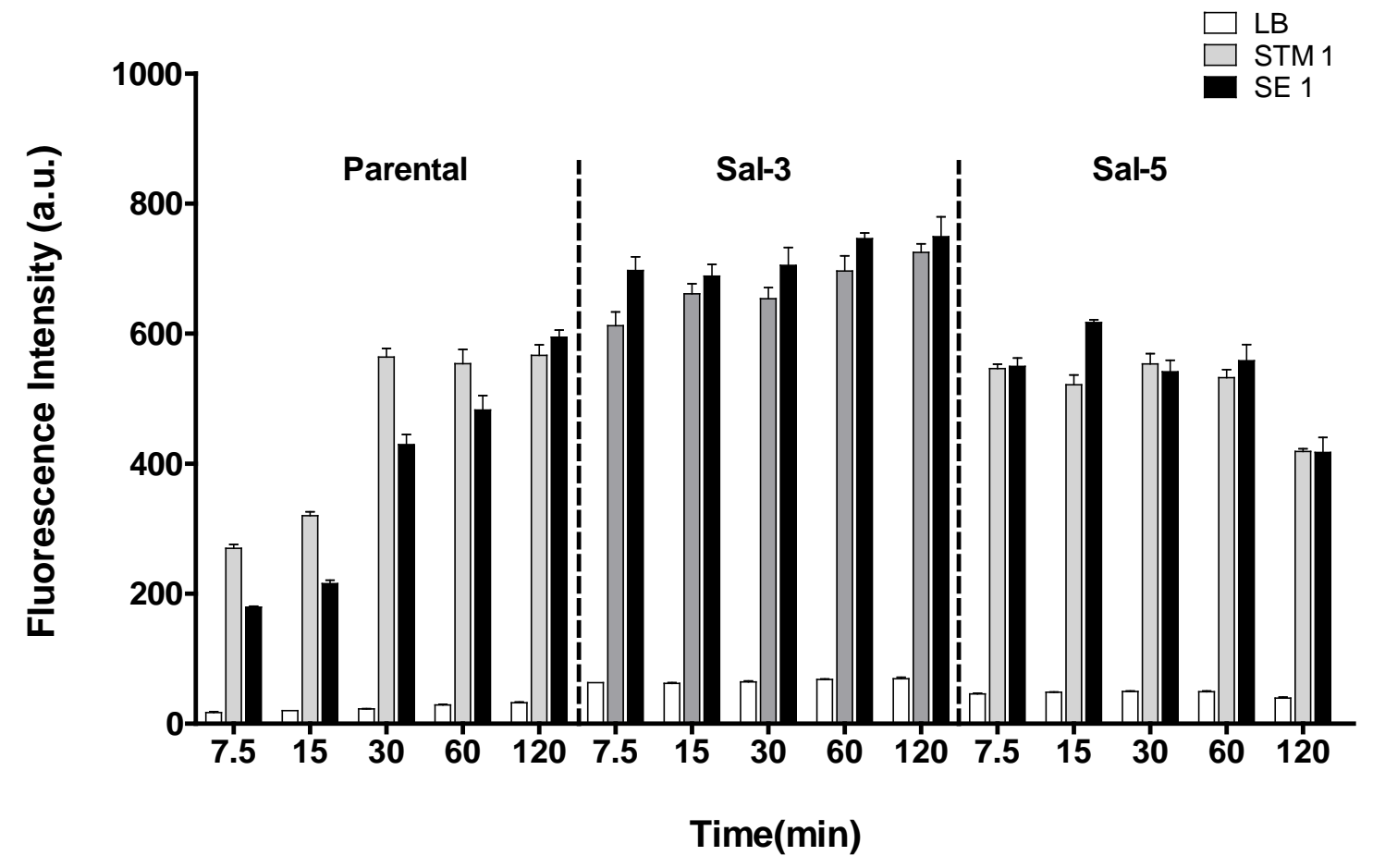

Figure S2a. Optimization of the incubation time for the Salmonella-specific oligonucleotide probes. 




Figure S2b. Chelation effect on the Salmonella-specific oligonucleotide probes. 


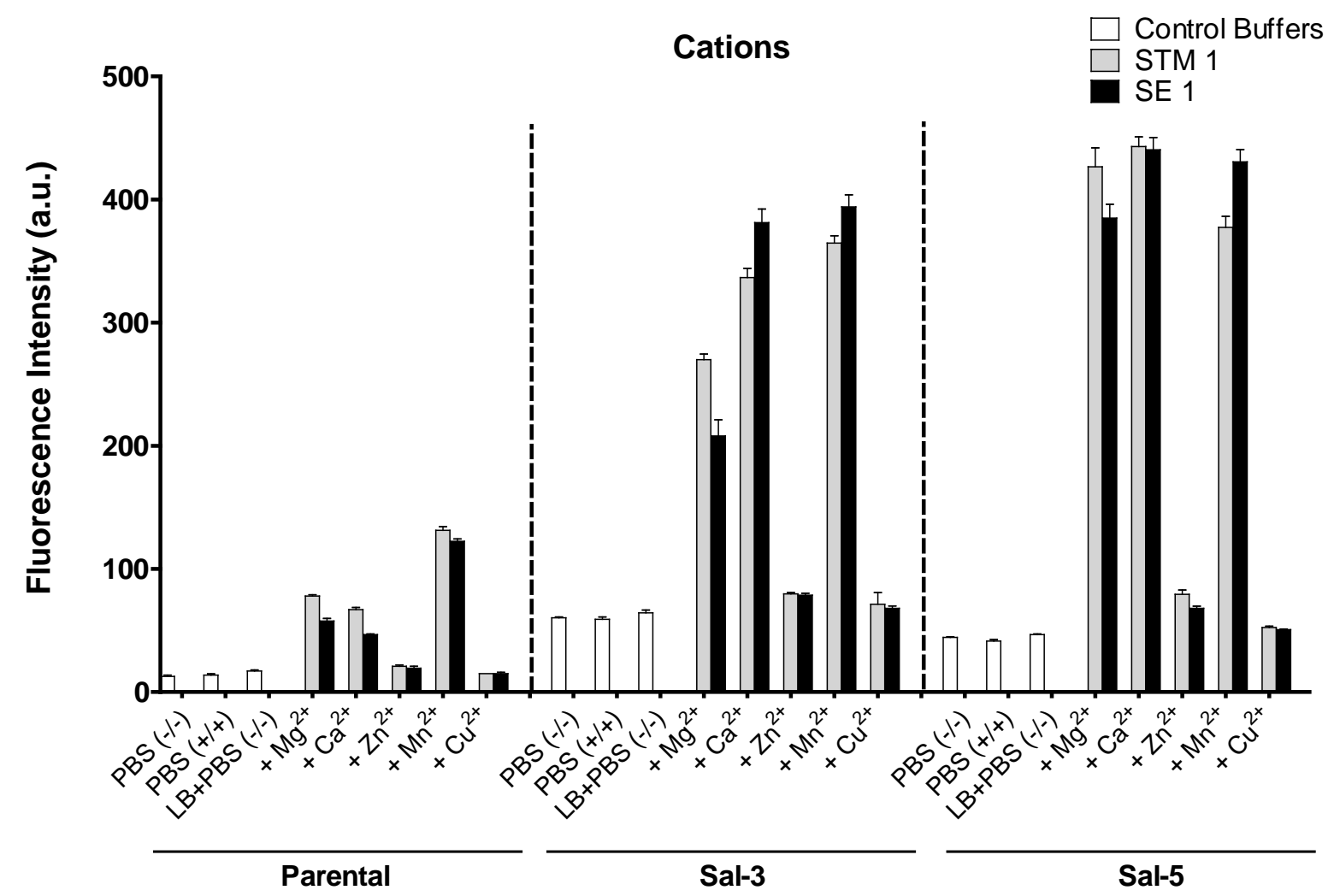

Figure S2c. Effect of the divalent cations on the Salmonella-specific oligonucleotide probes. 


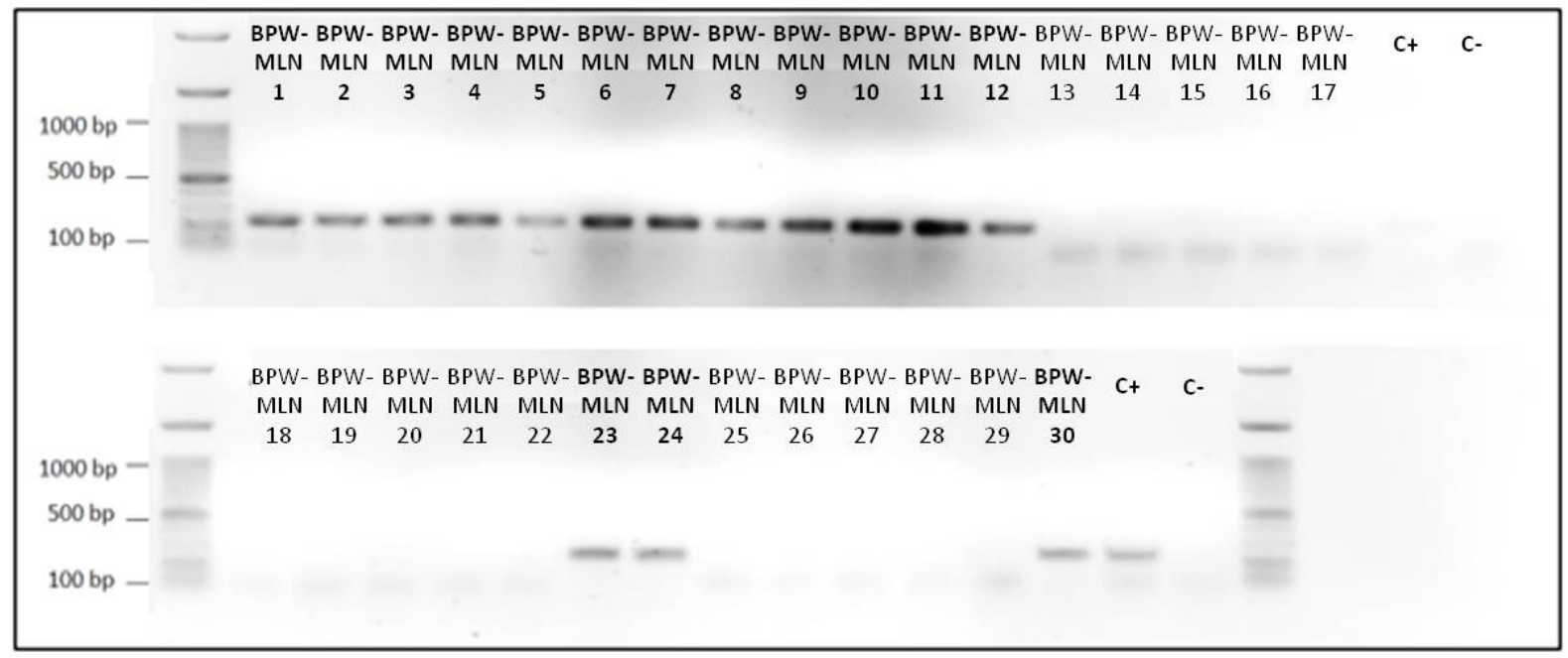

Figure S3. PCR of the lymph nodes samples (BPW-MLNnumber) to confirm the presence or absence of Salmonella. 
Table S3: Mesenteric lymph nodes samples and respective biochemical testing for the presence of Salmonella carried out by IdAB and the consequent determination of the Salmonella serotypes by the NRL. Table shows samples in the order they were analyzed.

\begin{tabular}{|c|c|c|}
\hline Name of sample & $\begin{array}{l}\text { Biochemical testing of } \\
\text { Salmonella (IdAB) }\end{array}$ & $\begin{array}{l}\text { Serotyping of Salmonella } \\
\text { (NRL) }\end{array}$ \\
\hline BPW-MLN1 & + & S. Typhimurium 1,4, [5],12:i:1,2 \\
\hline BPW-MLN2 & + & S. Typhimurium 1,4, [5],12:i:1,2 \\
\hline BPW-MLN3 & + & S. Typhimurium 1,4, [5],12:i:1,2 \\
\hline BPW-MLN4 & + & S. Typhimurium 1,4, [5],12:i:1,2 \\
\hline BPW-MLN5 & + & S. Typhimurium $1,4,[5], 12: \mathrm{i}: 1,2$ \\
\hline BPW-MLN6 & + & S. Typhimurium 1,4, [5],12:i:- \\
\hline BPW-MLN7 & + & S. Typhimurium 1,4, [5],12:i:1,2 \\
\hline BPW-MLN8 & + & S. Typhimurium $1,4,[5], 12: \mathrm{i}: 1,2$ \\
\hline BPW-MLN9 & + & S. Typhimurium 1,4, [5],12:i:1,2 \\
\hline BPW-MLN10 & + & S. arizonae 48:z4,z23,z32:- \\
\hline BPW-MLN11 & + & S. Derby 4,12:f,g:- \\
\hline BPW-MLN12 & + & S. Enteritidis 9,12:g,m:- \\
\hline BPW-MLN13 & - & \\
\hline BPW-MLN14 & - & \\
\hline BPW-MLN15 & - & \\
\hline BPW-MLN16 & - & \\
\hline BPW-MLN17 & - & \\
\hline BPW-MLN18 & - & \\
\hline BPW-MLN19 & - & \\
\hline BPW-MLN20 & - & \\
\hline BPW-MLN21 & - & \\
\hline BPW-MLN22 & - & \\
\hline BPW-MLN23 & + & S. Bardo 8:e,h:1,2 \\
\hline BPW-MLN24 & + & S. Typhimurium $1,4,[5], 12: \mathrm{i}: 1,2$ \\
\hline BPW-MLN25 & - & \\
\hline BPW-MLN26 & - & \\
\hline BPW-MLN27 & - & \\
\hline BPW-MLN28 & - & \\
\hline BPW-MLN29 & - & \\
\hline BPW-MLN30 & + & S. Typhimurium 1,4, [5],12:i:- \\
\hline
\end{tabular}


Sal-3

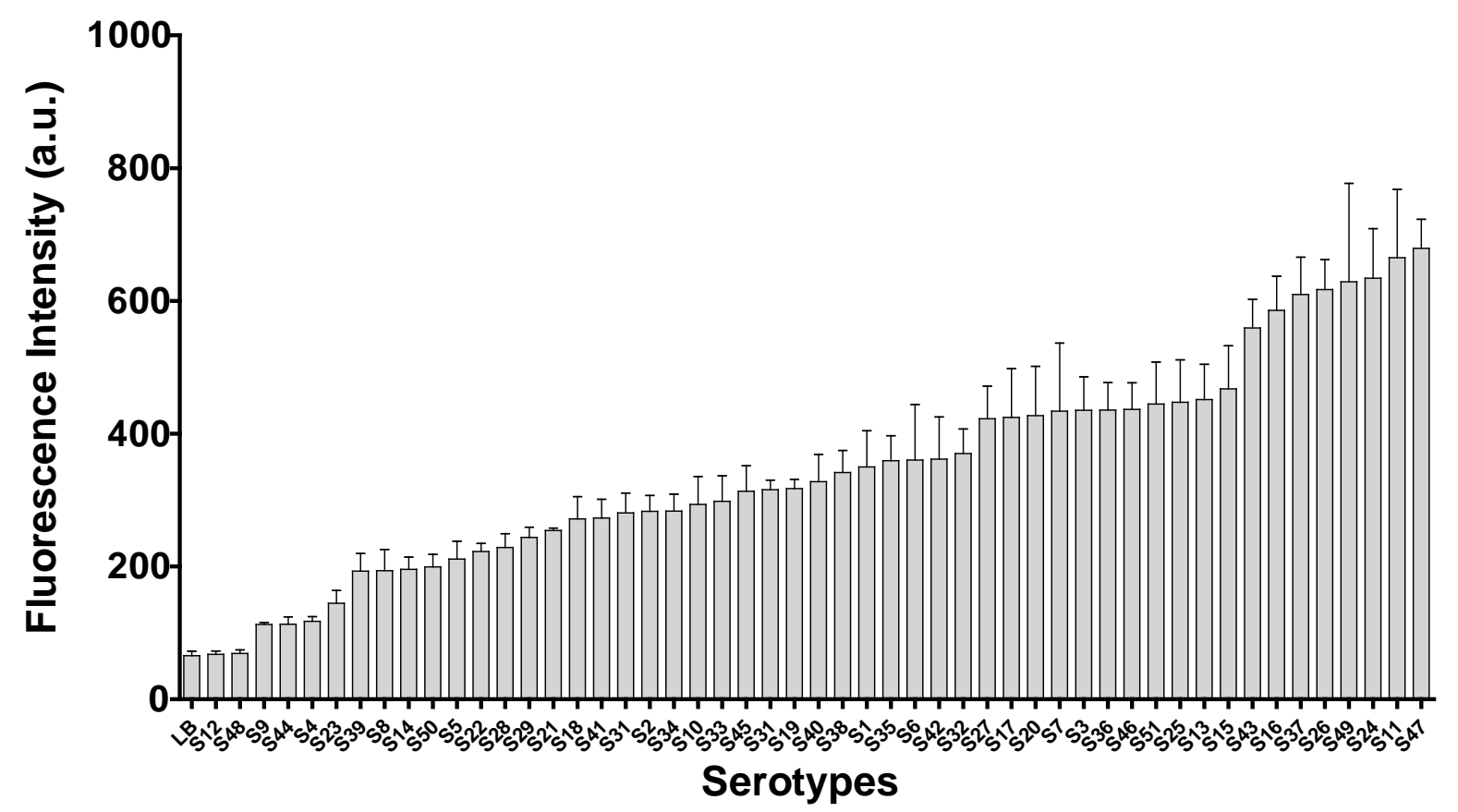

Figure S4a. Nuclease activity evaluation of 51 Salmonella serotypes using Sal-3 probe. Specific details for each serotype are reported in Table S1.

\section{Sal-5}

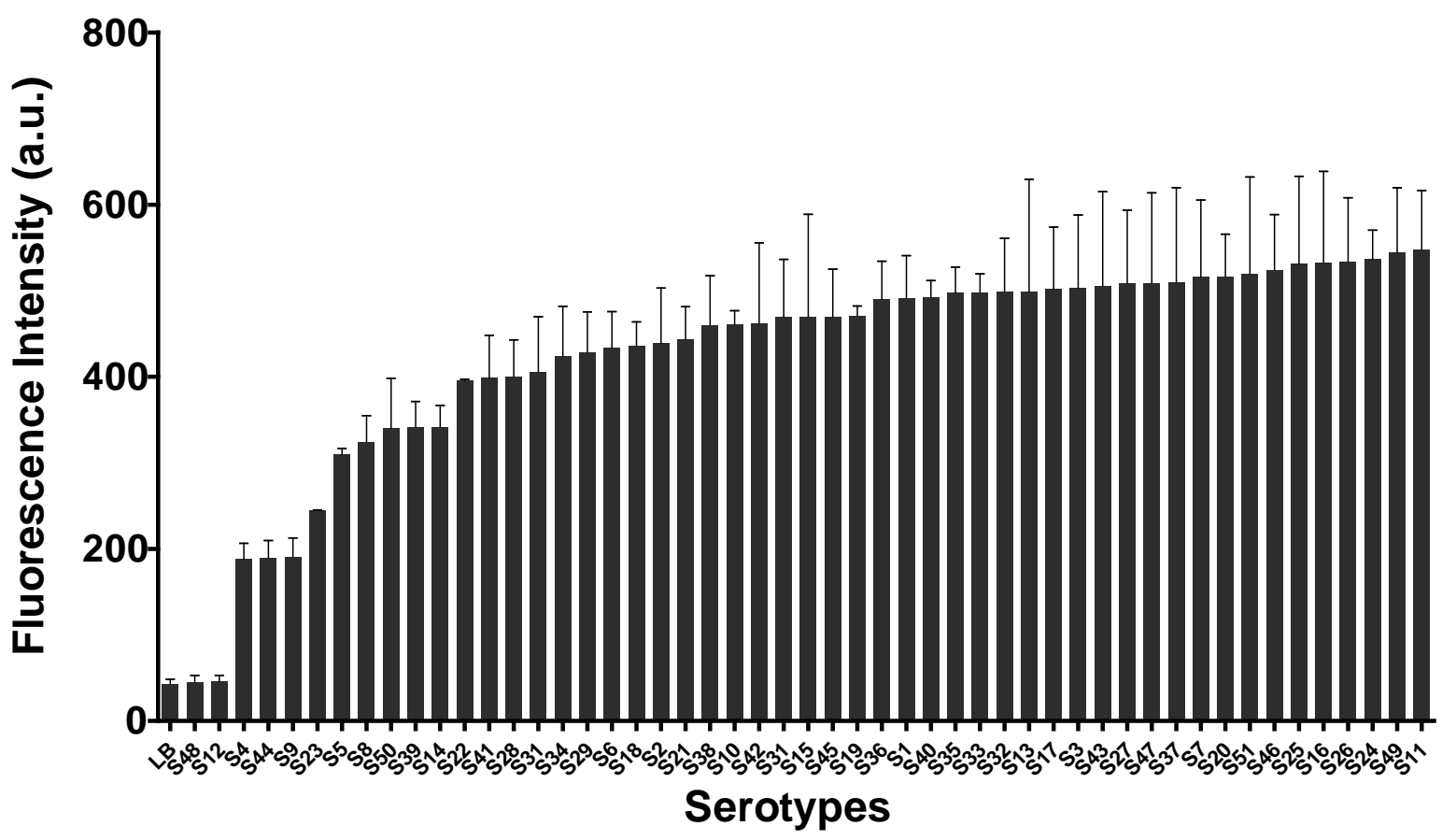

Figure S4b. Nuclease activity evaluation of 52 Salmonella serotypes using Sal-5 probe. Specific details for each serotype are reported in Table S1. 



Sal-3 (STM 1)



Sal-3 (SE 1)

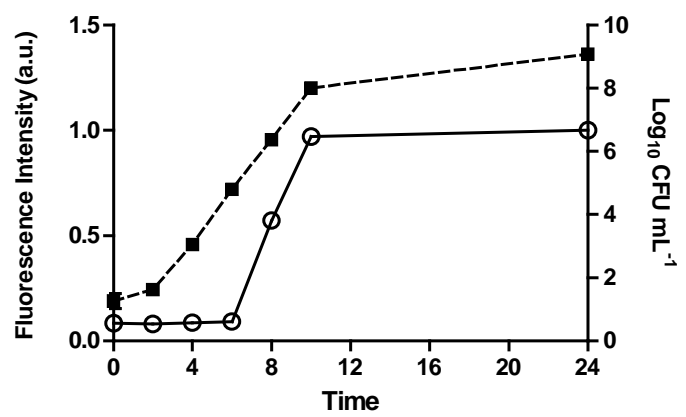

Sal-5 (STM 1)


- Nuclease activity

- Nuclease activity

$\rightarrow-\log _{10} \mathrm{CFU} \mathrm{mL}^{-1}$

$\rightarrow \log _{10} \mathrm{CFU} \mathrm{mL}$

Figure S5. Growth curves and nuclease activity of Salmonella cultures. Salmonella Typhimurium (STM 1) and Salmonella Enteritidis (SE 1) were cultured and CFU mL $\mathrm{mL}^{-1}$ determination at different time points was carried out by plate counting (black squares). The same samples (time points and cultures) were used for the evaluation in parallel of nuclease assay sensitivity for the Salmonella cultures (white circles). Fluorescence measurements of the nuclease activity derived from Salmonella Typhimurium (STM 1) and Salmonella Enteritidis (SE 1) were performed using the Parental, Sal-3 and Sal-5 probes at different time points. 
Table S4. Calculation of the LOD for STM 1 and SE 1 using nucleic acid probes.

STM 1

SE 1

\begin{tabular}{|l|ccc|ccc|}
\cline { 2 - 7 } \multicolumn{1}{l|}{ LOD $^{*}($ Sy $) *\left(\right.$ slope $\left.^{-1}\right)$} & Parental & Sal-3 & Sal-5 & Parental & Sal-3 & Sal-5 \\
\hline Sy & 0.2171 & 0.185 & 0.1602 & 0.2733 & 0.1777 & 0.1922 \\
Slope ${ }^{-1}$ & 11.29 & 9.749 & 8.16 & 11.95 & 7.72 & 7.277 \\
\left.${\text { LOD }\left(\log _{10} \text { CFU mL }\right.}^{-1}\right)$ & $\mathbf{7 . 3 5 3 1 7 7}$ & $\mathbf{5 . 4 1 0 6 9 5}$ & $\mathbf{3 . 9 2 1 6 9 6}$ & $\mathbf{9 . 7 9 7 8 0 5}$ & $\mathbf{4 . 1 1 5 5 3 2}$ & $\mathbf{4 . 1 9 5 9 1 8 2}$ \\
\hline
\end{tabular}

